Fakultät III

Wirtschaftswissenschaften, Wirtschaftsinformatik und Wirtschaftsrecht

Volkswirtschaftliche Diskussionsbeiträge

Discussion Papers in Economics

No. $171-14$

November 2014

Thomas Eichner · Rüdiger Pethig

Forging a global environmental agreement through trade sanctions on free riders? 
Universität Siegen

Fakultät III

Wirtschaftswissenschaften, Wirtschaftsinformatik und Wirtschaftsrecht

Fachgebiet Volkswirtschaftslehre

Hölderlinstraße 3

D-57068 Siegen

Germany

http://www.wiwi.uni-siegen.de/vwl/

ISSN 1869-0211

Available for free from the University of Siegen website at

http://www.wiwi.uni-siegen.de/vwl/research/diskussionsbeitraege/

Discussion Papers in Economics of the University of Siegen are indexed in RePEc and can be downloaded free of charge from the following website:

http://ideas.repec.org/s/sie/siegen.html 


\title{
Forging a global environmental agreement through trade sanctions on free riders?*
}

\author{
Thomas Eichner \\ Department of Economics, University of Hagen \\ Rüdiger Pethig \\ Department of Economics, University of Siegen
}

\begin{abstract}
This paper studies the formation of self-enforcing global environmental agreements in a world economy with international trade and two groups of countries that differ with respect to fuel demand and environmental damage. It investigates whether the signatories' threat to embargo (potential) free riders secures all countries' participation in the agreement. Resorting to numerical analysis, we find that an embargo may be unnecessary, ineffective or even counterproductive - depending on the degree of asymmetry and other parameters. On some subset of parameters, the embargo stabilizes the otherwise unstable global agreement, but the threat of embargo is not credible. However, in some of these cases credibility can be restored by suitable intra-coalition transfers.
\end{abstract}

JEL classification: F02, Q50, Q58

Key words: $\quad$ embargo, trade, asymmetry, free rider, fuel demand,

climate damage

*Eichner: Department of Economics, University of Hagen, Universitätsstr. 41, 58097 Hagen, Germany, email: thomas.eichner@fernuni-hagen.de; Pethig: Department of Economics, University of Siegen, Hölderlinstr. 3, 57068 Siegen, Germany, pethig@vwl.wiwi.uni-siegen.de. 


\section{The problem}

The reduction of global carbon emissions necessary to stabilize the world climate at safe levels requires a broad and deep international environmental agreement (IEA), i.e. an IEA that secures cooperation of all (major) countries and strives for maximum world welfare. The first legally binding IEA on climate change, the Kyoto Protocol, accomplished only little more than global non-cooperation. It expired in 2012, and the prospects are uncertain for reaching a more effective follow-up agreement. That calls for further efforts to improve our understanding of how to reach a broad and deep climate agreement.

Trade creates interdependence among countries in addition to the interdependence generated by global climate damage and is therefore important for understanding the (dis)incentives to cooperate. Although no sovereign countries can be forced to sign an IEA, it is possible to design an IEA that obliges all signatories to impose trade sanctions on non-signatories. ${ }^{1}$ The intention - or hope - is that the threat of sanctions suffices to induce participation of all countries. Among the few IEAs with trade sanctions to incentivize participation, is the Montreal Protocol. It bans trade between signatories and non-signatories in CFCs and other substances controlled by the treaty as well as imports from non-signatories of products containing these substances. Interestingly, participation in the Montreal Protocol turned out to be almost global without the need of actually implementing the ban. Van Slooten (1994), Brack (1996) and Barrett (2003) conclude that the ban had the intended effect of deterring countries from free riding. The present paper takes up the issue of trade sanctions to enhance further our understanding of how effective they are in forging broad and deep IEAs. We will disregard trade restrictions like import tariffs or border carbon adjustment ${ }^{2}$ but rather assume the strongest form of trade sanctions, a general embargo or trade ban threatened to be imposed on non-signatories by all signatories.

The analytical literature on IEAs with trade sanctions against free riders is small and offers mixed conclusions. Barrett (1997) studies a partial equilibrium model with symmetric countries where the countries play "abate" or "pollute" and imperfectly competitive firms sell a homogeneous product in segmented markets. He investigates how trade policy may help support self-enforcing ${ }^{3}$ IEAs and finds that the threat of trade sanctions is welfare

\footnotetext{
${ }^{1}$ Trade restrictions may not be compatible with WTO rules, Charnovitz (2007). In our paper, we ignore potential conflicts between environmentally motivated trade restrictions and international trade law.

${ }^{2}$ For various trade restrictions see Lessmann et al. (2009) and for border carbon adjustment see e.g. Elliott et al. (2010). The goal of this type of trade restrictions is primarily to level the playing field of international trade rather than inducing countries to participate in the IEA.

${ }^{3} \mathrm{An}$ IEA is said to be self-enforcing or a climate coalition is said to be stable, if no signatory has an incentive to defect from the IEA and no non-signatory has an incentive to sign. The literature on the formation of IEAs has adopted that stability concept from the theory of cartels (D'Aspremont et al. 1983).
} 
enhancing and may foster full participation. Chui Ying (2010) extends Barrett's (1997) approach to asymmetric countries and shows that economic sanctions could be credible, but cannot induce full participation of an IEA. In another game model, Barrett (1999) shows that a ban on trade is effective but not credible. ${ }^{4}$

In contrast to the studies of Barrett and Chui Ying, our point of departure is Eichner and Pethig $(2013,2014 a)$. They expand the basic model of the early literature on climate coalition formation ${ }^{5}$ by introducing world markets for fossil fuel and consumption goods ${ }^{6}$ and show for the case of identical countries, tax policy and international trade that the grand coalition is stable and implements the social optimum on some subset of parameters. Motivated by that remarkable result, Eichner and Pethig (2014b) expand the tax-policy model of Eichner and Pethig (2014a) by assuming two groups of countries that differ with respect to climate damage and the demand for energy. ${ }^{7}$ They find that asymmetric climate damage tends to destabilize and asymmetric energy demand tends to stabilize the grand coalition. The reason for the stabilizing effect of asymmetric energy demand is as follows. With full symmetry, trade takes place only between the coalition of all but one country and the outsider, but not between the (identical) coalition countries. With demand asymmetry, trade also takes place between coalition countries and thus makes it more attractive for the outsider to join. Asymmetry is also very important for the effectiveness of an embargo, because, in contrast to identical signatories, asymmetric signatories still enjoy gains from intra-coalition trade during the embargo, and therefore they suffer under the embargo less than identical signatories. Hence asymmetry promises to strengthen the incentives for full cooperation under the threat of embargo, and that is why the present paper investigates the consequences of the threat of embargo in the otherwise unchanged model of Eichner and Pethig (2014b). Having emphasized the relevance of asymmetry for coalition formation we

\footnotetext{
${ }^{4} \mathrm{~A}$ different strand of the literature, not followed here, employs large-scale simulation models. In such a model, Kempfert (2004) shows that trade sanctions do not necessarily induce outsiders to join a coalition. Lessmann et al. (2009) find a significant potential to raise participation and global welfare through import tariffs.

${ }^{5}$ Here we refer to the basic model introduced by Barrett (1994) and rigorously characterized by Diamantoudi and Sartzetakis (2006) and by Rubio and Ulph (2006). We consider corner solutions (e.g. zero emissions) an implausible artefact of the parametric approach and therefore follow Diamantoudi and Sartzetakis (2006) who restrict their focus on interior solutions.

${ }^{6}$ Eichner and Pethig (2013, 2014a, 2014b) place a game model on top of a simple general competitive equilibrium of the world economy, which increases analytical complexity considerably. The subsequent analysis also has to cope with this problem and does so by resorting to numerical calculations in cases of intractability.

${ }^{7}$ Since Barrett (1997) many asymmetric models (without trade) of self-enforcing IEAs have been studied with various simplifying assumptions and degrees of complexity, e.g. Carraro and Siniscalco (1998), Barrett (2001), McGinty (2007), Fuentes-Albero and Rubio (2010), Pavlova and de Zeeuw (2013).
} 
need to add that, from the viewpoint of theory, the downside of asymmetry is that it boosts complexity. The price to be paid is that we need to resort to numerical analysis.

In the present paper, we focus on the situation in which all countries but one have already signed a climate agreement that implements the social optimum, if all countries sign, and that obliges all signatories to impose a trade ban on free riders. The decision problem of the last and only outsider is then to sign or to stay outside. The outsider determines its positive or negative net benefit of signing assuming that all other countries stay in the coalition. ${ }^{8}$ It does sign the agreement, if it is better off signing than suffering under the embargo and if the coalition's threat of embargo is credible, i.e. if no signatory is worse off with than without imposing the embargo. If the threat of embargo is not credible and the outsider prefers free riding to joining in the absence of an embargo, then it will enjoy its free-rider position knowing that out of self-interest the signatories will not fulfill their obligation to embargo the free rider.

In our subsequent analysis, we distinguish three different regimes. The free-trade regime, in which no non-signatory is embargoed; the embargo regime, in which non-signatories face an embargo whether or not the threat of embargo is credible; and the threat-of-embargo regime, in which non-signatories are embargoed only, if the threat of embargo is credible. Ultimately, we are interested in the threat-of-embargo regime, of course. However, in order to obtain informative results about that regime we need to analyze and compare the other regimes first. Table 1 displays the possible outcomes of the transition from free trade to the embargo regime. Clearly, an effective embargo (box [2] in Table 1) is what we want an embargo to accomplish. However, somewhat unexpectedly we will have to deal with all boxes [1] - [4] of Table 1. Obviously, the credibility of the threat of embargo is not an issue when an embargo is unnecessary, counterproductive or ineffective (boxes [1], [3] or [4] in Table 1), because it is clear that in these cases the threat of embargo makes no sense in the first place.

\begin{tabular}{|c|c|c|c|}
\hline \multicolumn{4}{|c|}{ The grand coalition is stable } \\
\hline & & \multicolumn{2}{|c|}{$\ldots$ in the free-trade regime: } \\
\hline & & yes & no \\
\hline \multirow{2}{*}{... in the embargo regime: } & yes & [1] embargo unnecessary & [2] embargo effective \\
\hline & no & [3] embargo counterproductive & [4] embargo ineffective \\
\hline
\end{tabular}

Table 1: Comparison of outcomes in the scenario of free trade and embargo

\footnotetext{
${ }^{8}$ This crucial assumption is an implication of the stability concept (footnote 3 ). It portrays free riders as shortsighted and thus puts them in a favorable position.
} 
So we restrict the credibility check to those cases in which imposing an embargo stabilizes the otherwise unstable grand coalition (box [2] in Table 1). It turns out that one group of coalition countries is always worse off in the embargo regime than in the free-trade regime. We do not find conditions under which the embargo renders stable an otherwise unstable grand coalition and is credible, no matter how low or high the degree of asymmetry is. Given this negative result, we investigate whether it is possible through intra-coalition transfers to make all coalition countries better off in the embargo regime than in the freetrade regime. We find that such transfers can restore credibility, in fact, on a subset of the set of parameters that leads to the outcome of box [2].

In the following Section 2, we introduce some necessary notation and develop the analytical framework. Section 3 derives the countries' equilibrium welfares depending on parameters and asymmetries in the grand coalition and in the regimes of free trade and embargo. The central part of the paper is Section 4 that studies the conditions for the (in)stability of the grand coalition in five samples placing the focus on variations in the degree of asymmetry of climate damage (Samples 1-2) and of energy demand (Samples 3-5). Some parameters that are uniform across countries are also varied to improve the understanding of the (dis)incentives to cooperate. Section 6 investigates the possibility to make the threat of embargo credible through intra-coalition transfers. Section 7 concludes with an emphasis on the caveat concerning the robustness of the results.

\section{Analytical framework}

Following Eichner and Pethig (2014b), we consider a world economy with two groups of countries, group $M:=\{1, \ldots, m\}$ and group $N:=\{1, \ldots, n\}$ described by the following equations (1), (2) and (3): $:^{9,10}$

$$
\begin{aligned}
& x_{i}^{s}=X\left(e_{i}^{s}\right):=\bar{x}-\frac{\xi}{2}\left(e_{i}^{s}\right)^{2} \quad \text { for } i=1, \ldots, m+n, \\
& V^{m}\left(e_{i}^{d}\right)+x_{i}^{d}-D^{m}\left(\sum_{j=1}^{m+n} e_{j}^{d}\right)=a_{m} e_{i}^{d}-\frac{b}{2}\left(e_{i}^{d}\right)^{2}+x_{i}^{d}-\frac{\delta_{m}}{2}\left(\sum_{j=1}^{m+n} e_{j}^{d}\right)^{2} \quad \text { if } i \in M, \\
& V^{n}\left(e_{i}^{d}\right)+x_{i}^{d}-D^{n}\left(\sum_{j=1}^{m+n} e_{j}^{d}\right)=a_{n} e_{i}^{d}-\frac{b}{2}\left(e_{i}^{d}\right)^{2}+x_{i}^{d}-\frac{\delta_{n}}{2}\left(\sum_{j=1}^{m+n} e_{j}^{d}\right)^{2} \quad \text { if } i \in N, \\
& \sum_{j=1}^{m+n} x_{j}^{d}=\sum_{j=1}^{m+n} x_{j}^{s} \text { and } \sum_{j=1}^{m+n} e_{j}^{d}=\sum_{j=1}^{m+n} e_{j}^{s},
\end{aligned}
$$

\footnotetext{
${ }^{9}$ The superscripts $s$ and $d$ indicate quantities supplied and demanded, respectively. Upper-case letters denote functions. Subscripts attached to them indicate partial derivatives.

${ }^{10} \mathrm{We}$ use general functional forms such as $X$ in equation (1) only for convenience of notation. For reasons of tractability, we will make use of the linear-quadratic functional forms in (1) and (2) throughout the paper.
} 
where the parameters in (1) and (2) satisfy $\xi, \bar{x}, a_{m}, a_{n}, b, \delta_{m}, \delta_{n}>0$. According to the transformation function (1), each country $i=1, \ldots, m+n$ produces two goods, a standard composite consumption good (quantity $x_{i}^{s}$ ) and fossil energy (quantity $e_{i}^{s}$ ), called fuel. The function (1) is the same across countries and implies that both goods are produced by means of domestic productive factors with given endowments. To interpret the parameter $\xi$, observe that the costs (in terms of the consumption good) of extracting the amount $e_{i}^{s}$ of fossil fuel are

$$
\bar{x}-X\left(e_{i}^{s}\right)=\bar{x}-\left[\bar{x}-\frac{\xi}{2}\left(e_{i}^{s}\right)^{2}\right]=\frac{\xi}{2}\left(e_{i}^{s}\right)^{2},
$$

because $X\left(e_{i}^{s}\right)=\bar{x}$ for $e_{i}^{s}=0$ and $x_{i}^{s}=X\left(e_{i}^{s}\right)<\bar{x}$ for $e_{i}^{s}>0$. Therefore, $\xi$ is a measure of fuel extraction costs. On the macro level, it measures fossil fuel abundance or scarcity because for given prices total fuel output is strictly decreasing in $\xi$.

In each country $i$, a representative consumer demands the consumption good (quantity $x_{i}^{d}$ ) and fossil energy (quantity $e_{i}^{d}$ ) and suffers from climate damage. Her utility, also referred to as country $i$ 's welfare, is specified in equation (2) and depends on whether country $i$ belongs to the group of countries $M$ or $N$. For $i \in M, V^{m}\left(e_{i}^{d}\right)+x_{i}^{d}$ is the utility from consuming fuel and the consumption good. $D^{m}\left(\sum_{j=1}^{m+n} e_{j}^{d}\right)$ is the disutility or damage from climate change caused by aggregate carbon emissions. Carbon emissions are proportional to fuel consumption, and we therefore simply take $e_{i}^{d}$ to be both fuel demand and emissions. According to (2), consumers in both groups of countries may differ with respect to the parameters $a_{m}$ and $a_{n}$ or with respect to the parameters $\delta_{m}$ and $\delta_{n}$. For analytical convenience, we define $a_{m}:=a, a_{n}:=a+c, c \in \mathbb{R}$ and $\delta_{m}=\delta, \delta_{n}=\delta+\rho, \rho \in \mathbb{R}$ and place our main attention on exogenous variations of the asymmetry parameters $c$ and $\rho$ in what follows. Aggregate demand is required to match aggregate supply for fuel and the consumption good in the equations (3).

Eichner and Pethig (2014b) show that there exists a unique general competitive equilibrium with world markets for the consumption food (price $p_{x} \equiv 1$ ) and for fuel (producer price $p$ ), when each country regulates domestic emissions by means of an emission tax $t_{i}$. For the time being that tax is arbitrarily chosen. Defining $\mathbf{t}:=\left(t_{1}, \ldots, t_{m+n}\right)$, the equilibrium welfare of country $i$ reads

$$
\begin{array}{ll}
W^{m}(\mathbf{t}, c, \rho):=V^{m}\left(e_{i}^{d}\right)+X\left(e_{i}^{s}\right)+P(\mathbf{t}, c)\left(e_{i}^{s}-e_{i}^{d}\right)-D^{m}\left(\sum_{j=1}^{m+n} e_{j}^{d}\right) & \text { if } i \in M, \\
W^{n}(\mathbf{t}, c, \rho):=V^{n}\left(e_{i}^{d}\right)+X\left(e_{i}^{s}\right)+P(\mathbf{t}, c)\left(e_{i}^{s}-e_{i}^{d}\right)-D^{n}\left(\sum_{j=1}^{m+n} e_{j}^{d}\right) & \text { if } i \in N,
\end{array}
$$

where the equilibrium fuel price is

$$
p=P(\mathbf{t}, c):=\frac{\xi\left[(m+n) a+n c-\sum_{j=1}^{m+n} t_{j}\right]}{(m+n)(b+\xi)},
$$


and where $e_{i}^{s}$ and $e_{i}^{d}$ satisfy $e_{i}^{s}=\frac{P(\mathbf{t}, c)}{\xi}$ for $i \in M \cup N, e_{i}^{d}=\frac{\left[a-P(\mathbf{t}, c)-t_{i}\right]}{b}$ for $i \in M$ and $e_{i}^{d}=\frac{\left[a+c-P(\mathbf{t}, c)-t_{i}\right]}{b}$ for $i \in N$.

Note that due to the additivity of the utility function (2) the equilibrium allocation $\left(e_{i}^{d}, e_{i}^{s}, x_{i}^{d}, x_{i}^{s}\right)_{i \in M \cup N}$ depends on the fuel-demand asymmetry parameter $c$, but not on the damage asymmetry parameter $\rho$. The parameter $\rho$ affects the equilibrium welfare (4), however.

Central to our subsequent analysis is the concept of self-enforcing IEAs or stable environmental coalitions introduced by D'Aspremont et al. (1983), ${ }^{11}$ which requires that no signatory has an incentive to defect and no non-signatory has an incentive to sign. As pointed out in the Introduction, we restrict our attention to the stability of the grand coalition. It therefore suffices to compare the situation, in which a grand coalition exists, with situations with or without embargo consisting of a coalition of all countries but one, i.e. a coalition of $m+n-1$ countries, and a single country of group $M$ or $N$ outside the coalition. The outsider has to decide whether to join the coalition under the threat of embargo. Obviously, the outcome is a stable grand coalition, if and only if it is in the outsider's self-interest to join.

We denote as $f$-country (with $f=m, n$ ) the country of group $F=M, N$ outside the coalition of size $m+n-1$, and we call $h f$-country (with $h, f=m, n$ ) a country of group $H=M, N$ inside the coalition of size $m+n-1$ which faces an $f$-country of group $F=M, N$. When the $f$-country calculates its benefits from free riding, it anticipates whether the coalition will deliver on its threat of embargo. The $f$-country rightly expects an embargo, if and only if the coalition's threat is credible, i.e. if and only if all coalition countries are not worse off implementing the embargo than when they let the free rider go unpunished. If coalition countries are worse off, they will not live up to their threat of embargo, since there is no supranational enforcement agency to prevent them from following their self-interest. Hence, a threat that is not credible fails to deter free riding and renders unstable the grand coalition.

The complexity of the stability issue with the threat of embargo suggests distinguishing three different regimes: the free-trade regime, the embargo regime and the threat-of-embargo regime. The free-trade regime is the regime that prevails when the coalition refrains from imposing an embargo on the $f$-country. In the embargo regime, the $f$-country is embargoed, if it chooses to free ride, irrespective of whether the embargo makes coalition countries worse off. Finally, in the threat-of-embargo regime, the free-riding $f$-country is embargoed, if and only if the coalition countries are at least as well off after imposing the embargo as in the free-

\footnotetext{
${ }^{11}$ We use the terms "stable (environmental) coalition" and "self-enforcing IEA" synonymously.
} 
trade regime. Although our focus is on the threat-of-embargo regime, the thorough analysis of the regimes of free trade and embargo is a precondition for assessing the credibility of the threat of embargo.

\begin{tabular}{|c|c|c|c|}
\hline \multirow{3}{*}{$\begin{array}{l}\text { Welfare of the } \\
\downarrow\end{array}$} & \multicolumn{3}{|c|}{.. if the $f$-country } \\
\hline & ... does not join & he coalition in the & ... joins the coalition \\
\hline & embargo regime & free-trade regime & in either regime \\
\hline$f$-country, & $\hat{w}_{f}=\hat{\mathcal{W}}^{f}(c, \rho)$ & $\bar{w}_{f}=\overline{\mathcal{W}}^{f}(c, \rho)$ & $w_{f}^{*}=w_{h}^{*}=\mathcal{W}^{* h}(c, \rho)$ \\
\hline$h f$-country, & $\hat{w}_{h f}=\hat{\mathcal{W}}^{h f}(c, \rho)$ & $\bar{w}_{h f}=\overline{\mathcal{W}}^{h f}(c, \rho)$ & $w_{f}^{*}=w_{h}^{*}=\mathcal{W}^{* h}(c, \rho)$ \\
\hline
\end{tabular}

Table 2: Notation for the equilibrium welfares of the $f$-country and the $h f$-countries $(h, f=m, n)$ depending on regimes, on the $f$-country's decision to join or not to join the coalition, and on the asymmetry parameters $c$ and $\rho$

Table 2 introduces some notation we will use for the welfare of the $f$-country and the $h f$-countries in the regimes of free trade and embargo, when the $f$-country does or does not free ride. The functions $\mathcal{W}^{* h}, \overline{\mathcal{W}}^{f}, \overline{\mathcal{W}}^{h f}, \hat{\mathcal{W}}^{f}$ and $\hat{\mathcal{W}}^{h f}$ in Table 2 describe the dependence of equilibrium welfares on the degree of asymmetry that is measured by the asymmetry parameters $^{12} c$ and $\rho$. Taking advantage of that notation, the following statements hold: The grand coalition is stable

- in the free-trade regime, if and only if $w_{f}^{*} \geq \bar{w}_{f}$ for $f=m$ and $f=n$;

- in the embargo regime, if and only if $w_{f}^{*} \geq \hat{w}_{f}$ for $f=m$ and $f=n$;

- in the threat-of-embargo regime, if and only if $w_{f}^{*} \geq \hat{w}_{f}$ for $f=m$ and $f=n$ and $\hat{w}_{h f} \geq \bar{w}_{h f}$ for $h f=m m, m n, n m, n n$ (i.e., if and only if the grand coalition is stable in the embargo regime and the threat of embargo is credible).

\section{$3 \quad$ Equilibrium welfares}

\subsection{Welfares in the grand coalition and in the free-trade regime}

Eichner and Pethig (2014b) have derived the welfare functions of the second and third column of Table 2. It therefore suffices here to sketch the procedure. Obviously, the $f$-country's welfare in the grand coalition is independent of whether the free-trade regime or the embargo regime prevails. To derive the welfare function $\mathcal{W}^{* h}$, recall the welfare $W^{h}(\mathbf{t}, c, \rho)$ in (4) that represents the welfare countries of group $H=M, N$ attain in the competitive equilibrium

\footnotetext{
${ }^{12}$ We suppress all parameters other than the asymmetry parameters as arguments in these welfare functions, because our focus will be on systematic variations of the latter.
} 
with the tax profile $\mathbf{t}=\left(t_{1}, \ldots, t_{m+n}\right)$. An obvious necessary condition for maximizing the aggregate welfare of the grand coalition, as of all other coalitions, is that all members' tax rates be equal. Therefore, we can assign to all coalition countries the same tax rate, denoted $t_{z}$, and write the welfare of countries of group $H=M, N$ in the grand coalition as $W^{h}(\mathbf{t}, c, \rho)=W^{h}\left(t_{z}, c, \rho\right)$, with $\mathbf{t}=\underbrace{\left(t_{z}, \ldots, t_{z}\right)}_{(m+n) \text {-times }}$. Eichner and Pethig (2014b) show that the equilibrium welfare of countries of group $H$ is

$$
W^{h}\left(t_{z}, c, \rho\right):=V^{h}\left(e_{h}^{d}\right)+X\left(e_{h}^{s}\right)+P\left(t_{z}, c\right)\left(e_{h}^{s}-e_{h}^{d}\right)-D^{h}\left[(m+n) e_{h}^{s}\right] \quad \text { for } h=m, n,
$$

where $P\left(t_{z}, c\right):=\frac{\xi\left[(m+n)\left(a-t_{z}\right)+n c\right]}{(m+n)(b+\xi)}, e_{h}^{d}=\frac{a_{h}-P\left(t_{z}, c\right)-t_{z}}{b}$ and $e_{m}^{s}=e_{n}^{s}=\frac{P\left(t_{z}, c\right)}{\xi}$. Maximization of the grand coalition's aggregate welfare yields the socially optimal tax rate

$$
t_{z}^{*}=T(c, \rho):=\frac{[(m+n) a+n c][m \delta+n(\delta+\rho)]}{b+\xi+(m+n)[m \delta+n(\delta+\rho)]} .
$$

Insertion of $t_{z}^{*}$ from (7) in (6) finally yields

$$
w_{h}^{*}:=W^{h}\left(t_{z}^{*}, c, \rho\right)=W^{h}[T(c, \rho), c, \rho]=: \mathcal{W}^{* h}(c, \rho) \quad \text { for } h=m, n .
$$

Equation (8) specifies the function $\mathcal{W}^{* h}$ from Table 2.

Consider next the countries' welfare in the free-trade regime with a coalition of size $m+n-1$ and a free rider, $\overline{\mathcal{W}}^{f}(c, \rho)$ and $\overline{\mathcal{W}}^{h f}(c, \rho)$. We assign to each coalition country the tax rate $t_{z}$ and denote by $t_{f}$ the tax rate of the $f$-country, such that the $f$-country's equilibrium welfare is

$$
\overline{\mathcal{W}}^{f}\left(t_{f}, t_{z}, c, \rho\right):=V^{f}\left(e_{f}^{d}\right)+X\left(e^{s}\right)+\bar{P}\left(t_{f}, t_{z}, c\right)\left(e^{s}-e_{f}^{d}\right)-D^{f}\left[(m+n-1) e^{s}\right],
$$

and the $h f$-countries' equilibrium welfare is

$$
\bar{W}^{h f}\left(t_{f}, t_{z}, c, \rho\right):=V^{h}\left(e_{h f}^{d}\right)+X\left(e^{s}\right)+\bar{P}\left(t_{f}, t_{z}, c\right)\left(e^{s}-e_{h f}^{d}\right)-D^{h}\left[(m+n-1) e^{s}\right],
$$

where $\bar{P}\left(t_{f}, t_{z}, c\right):=\frac{\xi[(m+n) a+n c]-\left[t_{f}-(m+n-1) t_{z}\right]}{(m+n)(b+\xi)}, e_{f}^{d}=\frac{a_{f}-\bar{P}\left(t_{f}, t_{z}, c\right)-t_{f}}{b}, e_{f}^{s}=e_{m f}^{s}=e_{n f}^{s}=$ $\frac{\bar{P}\left(t_{f}, t_{z}, c\right)}{\xi}=: e^{s}$, and $e_{h f}^{d}=\frac{a_{h}-\bar{P}\left(t_{f}, t_{z}, c\right)-t_{z}}{b}$.

It follows that the aggregate welfare of the coalition of size $m+n-1$ is

$$
\bar{W}^{z f}\left(t_{f}, t_{z}, c, \rho\right):= \begin{cases}(m-1) \bar{W}^{m f}\left(t_{f}, t_{z}, c, \rho\right)+n \bar{W}^{n f}\left(t_{f}, t_{z}, c, \rho\right) & \text { if } f=m, \\ m \bar{W}^{m f}\left(t_{f}, t_{z}, c, \rho\right)+(n-1) \bar{W}^{n f}\left(t_{f}, t_{z}, c, \rho\right) & \text { if } f=n .\end{cases}
$$

The coalition and the $f$-country are players in a non-cooperative game with payoffs $\bar{W}^{z f}$ $\left(t_{f}, t_{z}, c, \rho\right), \bar{W}^{f}\left(t_{f}, t_{z}, c, \rho\right)$ and strategies $t_{z}$ and $t_{f}$. Our solution concept is the Nash equilibrium. That equilibrium is defined as a tuple of tax rates $\left[\bar{t}_{f}=\bar{T}^{f}(c, \rho), \bar{t}_{z f}=\bar{T}^{z f}(c, \rho)\right]$ satisfying $\bar{W}^{f}\left(\bar{t}_{f}, \bar{t}_{z f}, c, \rho\right) \geq \bar{W}^{f}\left(t_{f}, \bar{t}_{z f}, c, \rho\right)$ for all $t_{f}$ and $\bar{W}^{z f}\left(\bar{t}_{f}, \bar{t}_{z f}, c, \rho\right) \geq \bar{W}^{z f}\left(\bar{t}_{f}, t_{z}, c, \rho\right)$ 
for all $t_{z}$. Eichner and Pethig (2014b) calculate the Nash equilibrium tax rates and determine the Nash equilibrium welfares as

$$
\begin{aligned}
& \bar{w}_{f}:=\bar{W}^{f}\left(\bar{t}_{f}, \bar{t}_{z f}, c, \rho\right)=\bar{W}^{f}\left[\bar{T}^{f}(c, \rho), \bar{T}^{z f}(c, \rho), c, \rho\right]=: \overline{\mathcal{W}}^{f}(c, \rho), \\
& \bar{w}_{h f}:=\bar{W}^{h f}\left(\bar{t}_{f}, \bar{t}_{z f}, c, \rho\right)=\bar{W}^{h f}\left[\bar{T}^{f}(c, \rho), \bar{T}^{z f}(c, \rho), c, \rho\right]=: \overline{\mathcal{W}}^{h f}(c, \rho) .
\end{aligned}
$$

Thus we have substantiated the functions $\overline{\mathcal{W}}^{f}$ and $\overline{\mathcal{W}}^{h f}$ in Table 2.

\subsection{Welfares in the embargo regime with a free rider}

Conceptually, we follow the procedure of Section 3.1 to specify the welfare functions $\hat{\mathcal{W}}^{f}$ and $\hat{\mathcal{W}}^{h f}$ introduced in Table 2. As before, we denote the coalition countries' tax rate by $t_{z}$ and the $f$-country's tax rate by $t_{f}$. Accordingly, in the non-cooperative competitive equilibrium with tax rates $t_{z}$ and $t_{f}$ the $f$-country's welfare now is $\hat{W}^{f}\left(t_{f}, t_{z}, c, \rho\right)$ and the coalition countries' welfare is $\hat{W}^{h f}\left(t_{f}, t_{z}, c, \rho\right)$. To specify $\hat{W}\left(t_{f}, t_{z}, c, \rho\right)$, we consider the trade embargo and readily derive for the $f$-country's autarkic economy, $p_{f}=\frac{\xi\left(a_{f}-t_{f}\right)}{b+\xi}, e_{f}^{s}=\frac{p_{f}}{\xi}$, $e_{f}^{d}=\frac{a_{f}-p_{f}-t_{f}}{b}$, and $e_{f}^{s}=e_{f}^{d}=\frac{a_{f}-t_{f}}{b+\xi}$. Hence,

$$
\hat{W}^{f}\left(t_{f}, t_{z}, c, \rho\right)=V^{f}\left(e_{f}^{d}\right)+X\left(e_{f}^{s}\right)-D^{f}\left[e_{f}^{s}+(m+n-1) e_{z f}^{s}\right],
$$

where $e_{z f}^{s}$ is the coalition countries' fuel supply, when the outside country belongs to group $F$. To specify that supply, we proceed as follows. In view of $e_{h f}^{s}=\frac{p_{z f}}{\xi}=: e_{z f}^{s}$ for $h, f=m, n$, $e_{h f}^{d}=\frac{a_{h}-p_{z f}-t_{z}}{b}$ for $h=m, n$ and the condition for clearing the intra-coalition fuel market we calculate the equilibrium fuel price as

$$
p_{z f}=\hat{P}^{f}\left(t_{z}, c\right):= \begin{cases}\frac{\xi\left[(m+n-1)\left(a-t_{z}\right)+n c\right]}{(m+n-1)(b+\xi)} & \text { if } f=m \\ \frac{\xi\left[(m+n-1)\left(a-t_{z}\right)+(n-1) c\right]}{(m+n-1)(b+\xi)} & \text { if } f=n .\end{cases}
$$

Plugging $\hat{P}^{f}\left(t_{z}, c\right)$ into the coalition countries' fuel demands and supplies yields

$$
e_{h f}^{d}=\frac{a_{h}-\hat{P}^{f}\left(t_{z}, c\right)-t_{z}}{b} \quad \text { and } \quad e_{z f}^{s}=\frac{\hat{P}^{f}\left(t_{z}, c\right)}{\xi} .
$$

This information allows specifying the individual and aggregate welfares in the coalition as

$$
\begin{aligned}
& \hat{W}^{h f}\left(t_{f}, t_{z}, c, \rho\right):=V^{h}\left(e_{h f}^{d}\right)+X\left(e_{z f}^{s}\right)+\hat{P}^{f}\left(t_{z}, c\right)\left(e_{z f}^{s}-e_{h f}^{d}\right)-D^{h}\left[e_{f}^{s}+(m+n-1) e_{z f}^{s}\right], \\
& \hat{W}^{z f}\left(t_{f}, t_{z}, c, \rho\right):=\left\{\begin{array}{l}
(m-1) \hat{W}^{m f}\left(t_{f}, t_{z}, c, \rho\right)+n \hat{W}^{n f}\left(t_{f}, t_{z}, c, \rho\right) \text { if } f=m, \\
m \hat{W}^{m f}\left(t_{f}, t_{z}, c, \rho\right)+(n-1) \hat{W}^{n f}\left(t_{f}, t_{z}, c, \rho\right) \text { if } f=n .
\end{array}\right.
\end{aligned}
$$

As in the free-trade regime, the coalition and the $f$-country are players in a non-cooperative game. Their payoffs are $\hat{W}^{z f}\left(t_{f}, t_{z}, c, \rho\right)$ and $\hat{W}^{f}\left(t_{f}, t_{z}, c, \rho\right)$ from (13) and (15) and their 
strategies are $t_{z}$ and $t_{f}$, respectively. In the Appendix A we calculate the Nash equilibrium tax rates. Inserting these tax rates in (13) and (15) yields the Nash equilibrium welfares,

$$
\begin{aligned}
\hat{w}_{f} & :=\hat{W}^{f}\left(\hat{t}_{f}, \hat{t}_{z f}, c, \rho\right)=\hat{W}^{f}\left[\hat{T}^{f}(c), \hat{T}^{z f}(c), c, \rho\right]:=\hat{\mathcal{W}}^{f}(c, \rho), \\
\hat{w}_{h f} & :=\hat{W}^{h f}\left(\hat{t}_{f}, \hat{t}_{z f}, c, \rho\right)=\hat{W}^{h f}\left[\hat{T}^{f}(c), \hat{T}^{z f}(c), c, \rho\right]:=\hat{\mathcal{W}}^{h f}(c, \rho) .
\end{aligned}
$$

The equations (16) specify the functions $\hat{\mathcal{W}}^{f}$ and $\hat{\mathcal{W}}^{h f}$ in Table 2.

\section{Size and asymmetry of climate damage and the threat of embargo $(\delta \uparrow \downarrow, \rho \uparrow \downarrow, c=0)$}

Throughout this section, we assume that consumer preferences for fuel are the same in all countries $(c=0)$, whereas the climate damage may differ across the groups of countries $(\rho \neq 0)$. The first step towards assessing the impact of the threat of embargo on free riding is to investigate the conditions under which the grand coalition is stable in the embargo regime.

\subsection{Stability of the grand coalition in the embargo regime}

The grand coalition is stable [instable] in the embargo regime, if the welfare difference $\hat{\mathcal{D}}^{f}(\rho):=\mathcal{W}^{* h}(\rho)-\hat{\mathcal{W}}^{f}(\rho)$ is non-negative [negative] for $f=h=m, n{ }^{13}$ The question we are interested in is whether in the embargo regime the conditions for the stability of the grand coalition improve, when the climate damage differs across groups $(\rho \neq 0)$. Since analytical complexity prevents the derivation of informative general results, we turn to numerical analysis and investigate the stability of the grand coalition in the Samples 1 and 2 of Table 3. These samples differ only with respect to the size of the parameter $\delta$ and include the continuous variation of the damage parameter $\delta$ in the interval ${ }^{14}\left[\rho_{\min }, \rho_{\max }\right]$, which is the set of all values of $\delta$, for which the equilibrium demands and supplies of fuel are positive. ${ }^{15}$

\footnotetext{
${ }^{13}$ To avoid clutter, we write $\hat{\mathcal{D}}^{f}(\rho)$ short for $\hat{\mathcal{D}}^{f}(c=0, \rho)$ etc. in the present section.

${ }^{14}$ For details see Appendix B. We keep our analysis within that interval, because negative quantities are an artefact of the linear-quadratic functional forms (1) and (2), and zero consumption of fuel is highly unrealistic. Positive values of the demand and supply of the consumption good are secured by choosing a sufficiently high value of the parameter $\bar{x}$. To avoid clutter, we write $\left[\rho_{\min }, \rho_{\max }\right]$ instead of $\left[\rho_{\min }(\delta), \rho_{\max }(\delta)\right]$. Also, we set $\rho_{\min }(\delta)=-\delta$ and thus avoid dealing with climate change benefits (= negative climate damage).

${ }^{15} \mathrm{We}$ use the term sample rather than example because each sample characterizes the outcome along the asymmetry dimension $\rho$ and hence consists of a continuum of examples.
} 


\begin{tabular}{|c|c|c|c|c|c|c|c|}
\cline { 2 - 8 } \multicolumn{1}{c|}{} & $\delta_{m}=\delta$ & $a_{m}=a$ & $m=n$ & $b$ & $\xi$ & $\rho=\delta_{n}-\delta$ & $c=a_{n}-a$ \\
\hline \hline Sample 1 & 1,600 & 100 & 5 & 2,000 & 100,000 & $\in\left[\rho_{\min }, \rho_{\max }\right]$ & 0 \\
\hline Sample 2 & 3 & 100 & 5 & 2,000 & 100,000 & $\in\left[\rho_{\min }, \rho_{\max }\right]$ & 0 \\
\hline
\end{tabular}

Table 3: Degree of asymmetry and level of climate damage: Samples 1 and 2

Figure 1 determines for Sample 1 the sign of the welfare difference $\hat{\mathcal{D}}^{f}(\rho)=\mathcal{W}^{* h}(\rho)-$ $\hat{\mathcal{W}}^{f}(\rho)<0$ for $f=h=m, n$ and for all $\rho \in\left[\rho_{\min }, \rho_{\max }\right]$, where $\rho_{\min }=-1,600$ and $\rho_{\max }=1,596$. The straightforward result is that a country of either group prefers being embargoed to joining the coalition on the entire domain of asymmetry, $\left[\rho_{\min }, \rho_{\max }\right]$. Hence, under the conditions of Sample 1 an embargo fails to forge a stable grand coalition.
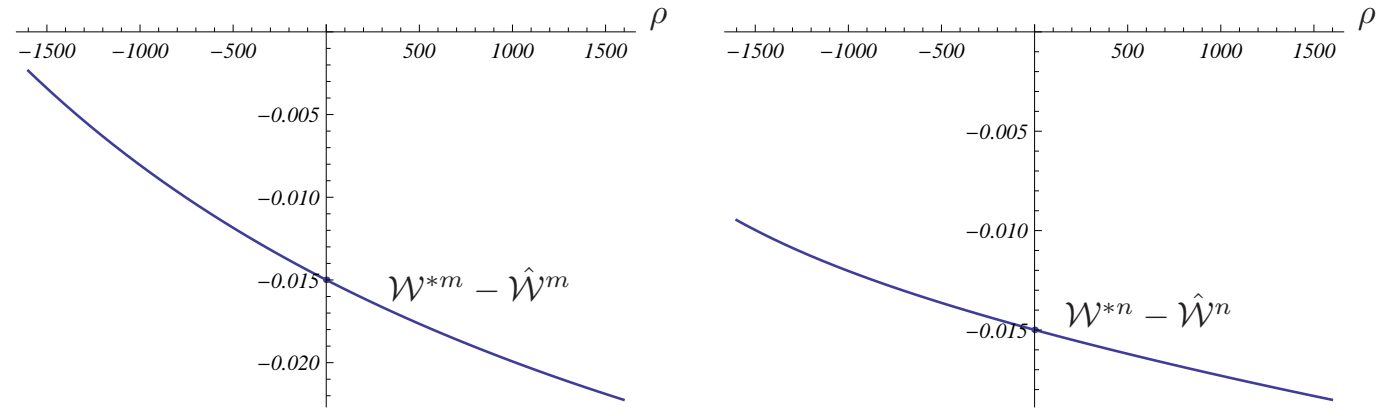

Figure 1: Welfare difference functions $\hat{\mathcal{D}}^{m}(\rho)$ and $\hat{\mathcal{D}}^{n}(\rho)$ in Sample $1(\delta=1,600)$

In Sample 2 the boundary points of the feasibility interval $\left[\rho_{\min }, \rho_{\max }\right]$ are $\rho_{\min }=-3$ and $\rho_{\max }=4,429$. Figure 2 shows that in Sample 2 we get the same qualitative result as in Sample 1: $\hat{\mathcal{D}}^{f}(\rho)<0$ for all $f=m, n$ and for all $\rho \in\left[\rho_{\min }, \rho_{\max }\right]$. Hence, under the conditions of Sample 2 an embargo also fails to forge a stable grand coalition.

\subsection{Credibility of the threat of embargo}

The negative result of Section 4.1 clearly disqualifies the embargo policy as an instrument to stabilize the grand climate coalition in the Samples 1 and 2 that focus on asymmetric climate damage. In particular, there is no sense in examining the credibility of the threat of embargo, since the desired result of an embargo, namely the stabilization of the grand coalition, cannot be secured - irrespective of whether the threat of embargo is credible. Nonetheless, we will briefly investigate by means of the Tables 4 and 5 how the embargo 

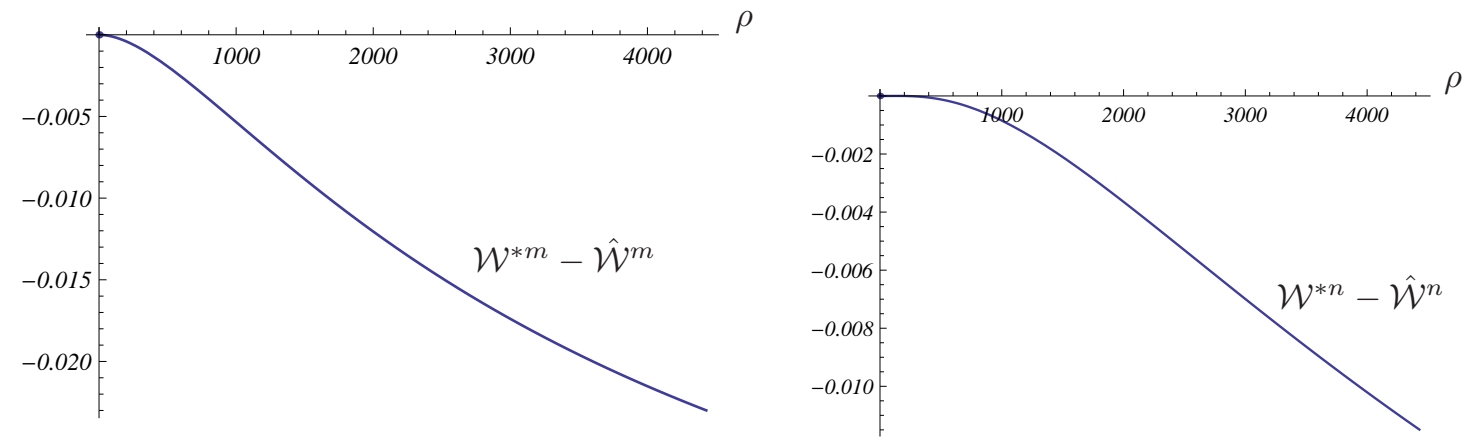

Figure 2: Welfare difference functions $\hat{\mathcal{D}}^{m}(\rho)$ and $\hat{\mathcal{D}}^{n}(\rho)$ in Sample $2(\delta=3)$

regime compares with the free-trade regime in case of the Samples 1 and 2. The first row of these tables presents the results from Section 4.1 and the third row contains the corresponding information "imported" from Eichner and Pethig (2014b). ${ }^{16}$

\begin{tabular}{|c|c|}
\hline Embargo regime & unstable \\
\hline Free-trade regime & unstable \\
\hline Parameter $\rho \rightarrow$ & $\rho=0$ \\
\hline
\end{tabular}

Table 4: Stability in the regimes of free trade and embargo in Sample $1(\delta=1,600)$

\begin{tabular}{|l||c|c|c|}
\hline Embargo regime & \multicolumn{4}{|c|}{ unstable } \\
\hline \hline Sub-interval no.: & 1 & 2 & 3 \\
\hline \hline Free-trade regime & unstable & stable & unstable \\
\hline \hline & & $\vdots$ & $\bar{\rho}_{m}$
\end{tabular}

Table 5: Stability in the regimes of free trade and embargo in Sample $2(\delta=3)$

For the case of high climate damage (Sample 1), the comparison of regimes in Table

\footnotetext{
${ }^{16}$ Eichner and Pethig (2014b) study the Samples 1 and 2 as specified in Table 3 in the free-trade regime. Here we only present their results and refer the reader to Eichner and Pethig (2014b) for further discussion and interpretation of these results. We will apply the same "import" procedure below for the Samples 3,4 and 5 of Table 4 .
} 
4 shows that an embargo is ineffective (box [4] in Table 1) with and without asymmetric climate damage. If the climate damage is low (Sample 2), an embargo is also ineffective in the sub-intervals 1 and 3 of Table 5 with high climate damage asymmetry $|\rho|$. However, there is an interval $\left[\bar{\rho}_{n}, \bar{\rho}_{m}\right]$ containing $\rho=0$ in which the grand coalition is stable in the free-trade regime but unstable in the embargo regime. In that case implementing an embargo would be counterproductive (box [3] in Table 1), because it would destabilize the otherwise stable grand coalition.

The explanation for the puzzle of Table 5 that in the interval $\left[\bar{\rho}_{m}, \bar{\rho}_{n}\right]$ an embargo destabilizes the otherwise stable grand coalition is as follows. According to Eichner and Pethig (2014a) the grand coalition is stable in the free-trade regime when the parameters of Sample 2 are given and all countries are alike $(c=0)$. If the coalition implements an embargo in that case of full symmetry, not only the free rider is pushed into the state of autarky, but international trade also ceases within the coalition of size $m+n-1$ because all countries in that coalition are alike. Hence, the embargo turns the world economy from trade between the free rider and the coalition into full autarky in which the grand coalition is known to be unstable (Eichner and Pethig 2014a). As the third row of Table 5 shows, the incentives for cooperation tend to decline with growing asymmetry in the free-trade regime, ${ }^{17}$ and this is also true in the embargo regime. Since the grand coalition is unstable for $c=0$ in the embargo regime of the Samples 1 and 2, increasing asymmetry fails to turn that result around.

One can show that the negative results of the Samples 1 and 2 not only hold for the parameter values $\delta=1,600$ (Sample 1) and $\delta=3$ (Sample 2), but also for all $\delta>0$. If we vary the damage parameter $\delta>0$ continuously and keep unchanged all other parameters in Table 3, we find that in qualitative terms the outcome is the same as in the Figures 1 and 2 for all $\delta>0$. We summarize these findings in

Result 1. (Embargo and size and asymmetry of climate damage)

Set the parameters $a=100, b=2,000, c=0, m=n=5$ and $\xi=100,000$ that are common to the Samples 1 and 2 and consider all feasible pairs $(\delta, \rho)$. Threatening to embargo free riders is not suitable as a policy to forge the grand climate coalition for any pair $(\delta, \rho)$, because for all $\delta>0$, the grand coalition is unstable in the entire interval $\left[\rho_{\min }(\delta), \rho_{\max }(\delta)\right]$ of feasible climate damage asymmetries.

\footnotetext{
${ }^{17}$ For more details see Eichner and Pethig (2014b)
} 


\section{Size and asymmetry of fuel demand, size of extraction costs and the threat of embargo $(a \uparrow \downarrow, c \uparrow \downarrow, \rho=0, \xi \uparrow \downarrow)$}

Now we assume that the climate damage hits all countries in the same way $(\rho=0)$, whereas the preferences for fuel may differ across countries $(c \neq 0)$. As in Section 4 , the complexity of the asymmetry analysis forces us into numerical analysis. Specifically, we will explore the impact on the stability of the grand coalition of continuous variations of the fuel-demand asymmetry parameter $c$ by means of the Samples 3, 4 and 5 specified in Table 6 . These samples differ with respect to the parameter $\xi$ only and in each sample, the parameter $c$ will be varied in the interval ${ }^{18}\left[c_{\min }, c_{\max }\right]$, the set of all values of $c$, for which the equilibrium demands and supplies of fuel are positive. Sample 5 equals Sample 1 except that we turn the asymmetry $\rho \neq 0$ and $c=0$ from Sample 1 into the asymmetry $c \neq 0$ and $\rho=0$ in Sample 5.

\begin{tabular}{|c||c|c|c|c|c|c|c|}
\cline { 2 - 8 } & $\delta_{m}=\delta$ & $a_{m}=a$ & $m=n$ & $b$ & $\xi$ & $\rho=\delta_{n}-\delta$ & $c=a_{n}-a$ \\
\hline \hline Sample 3 & 3 & $100^{*)}$ & 5 & 2,000 & 5,000 & 0 & $\in\left[c_{\min }, c_{\max }\right]$ \\
\hline Sample 4 & 3 & 100 & 5 & 2,000 & 500 & 0 & $\in\left[c_{\text {min }}, c_{\text {max }}\right]$ \\
\hline Sample 5 & 3 & 100 & 5 & 2,000 & 100,000 & 0 & $\in\left[c_{\text {min }}, c_{\text {max }}\right]$ \\
\hline
\end{tabular}

*) For the case of Sample 3, Section 5.1 also studies variations of parameter $\alpha$

Table 6: Fuel preference asymmetry and extraction costs: Sample 3, 4 and 5

Section 5 is organized as follows. Section 5.1 investigates Sample 3 and determines $\hat{\mathcal{D}}^{f}(\cdot)$ first for all fuel-demand asymmetry parameters $c \in\left[c_{\min }, c_{\max }\right]$ and then for the set of all feasible tuples $(a, c)$. The continuous variation of the parameter $a$ serves to study the interdependence of the impacts of the size and asymmetry of fuel demand. For the Samples 3,4 and 5, the first part of Section 5.2 determines the sign of the welfare difference $\hat{\mathcal{D}}^{f}(c)$, $f=m, n$, on the interval $\left[c_{\min }, c_{\max }\right]$ and examines the credibility of the threat of embargo. Here we keep the parameter $a$ constant at $a=100$, because conceptually the effects of its variations are as in Sample 3. However, we expand the Samples 3, 4 and 5 along the extraction costs dimension, $\xi$, that is the only dimension in which the Samples 3, 4 and 5 differ.

\footnotetext{
${ }^{18}$ The feasibility interval $\left[c_{\min }, c_{\max }\right]$ is the analogue to the interval $\left[\rho_{\min }, \rho_{\max }\right]$ in Section 4 . To avoid clutter, we write $\left[c_{\min }, c_{\max }\right]$ instead of $\left[c_{\min }(\xi), c_{\max }(\xi)\right]$.
} 


\subsection{Size and asymmetry of fuel demand $(c \uparrow \downarrow, a \uparrow \downarrow, \rho=0)$}

In the Figures 3 and 4 we determine for Sample 3 the sign of the welfare differences $\hat{\mathcal{D}}^{m}(c)$ and $\hat{\mathcal{D}}^{n}(c)$ for all $c \in\left[c_{\min }, c_{\max }\right]$. The right-hand-side panels of these figures depict enlarged segments of the welfare difference curves, which enables us to identify the (sign of the) intersection points $\hat{c}_{m 1}$ and $\hat{c}_{m 2}$, respectively.
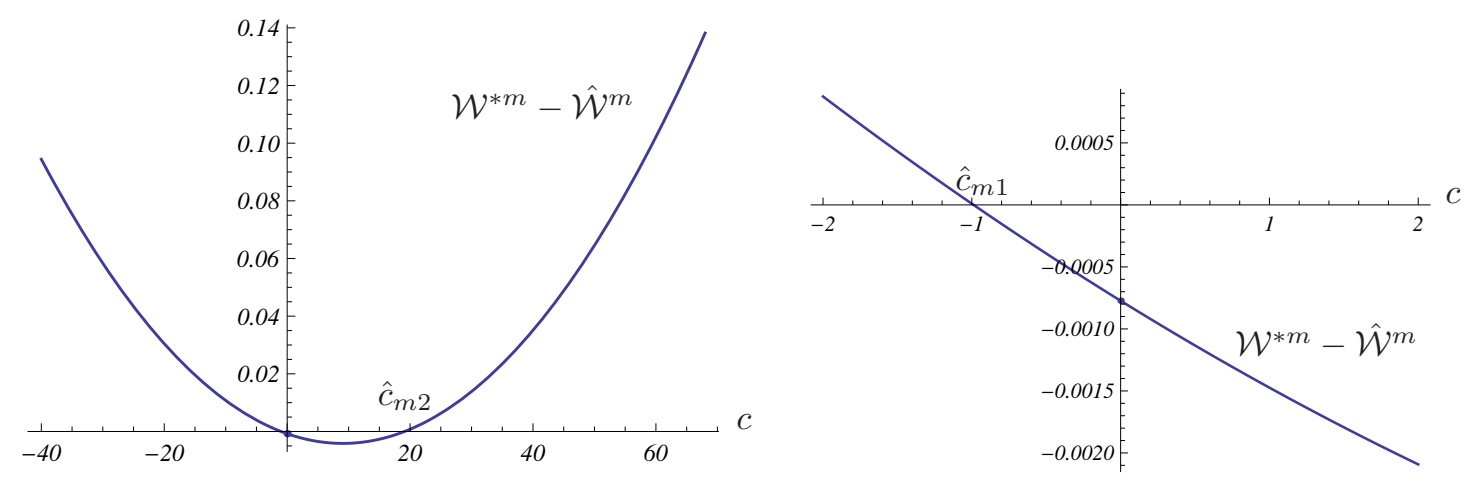

Figure 3: Welfare difference function $\hat{\mathcal{D}}^{m}$ in Sample $3(\xi=5,000)$
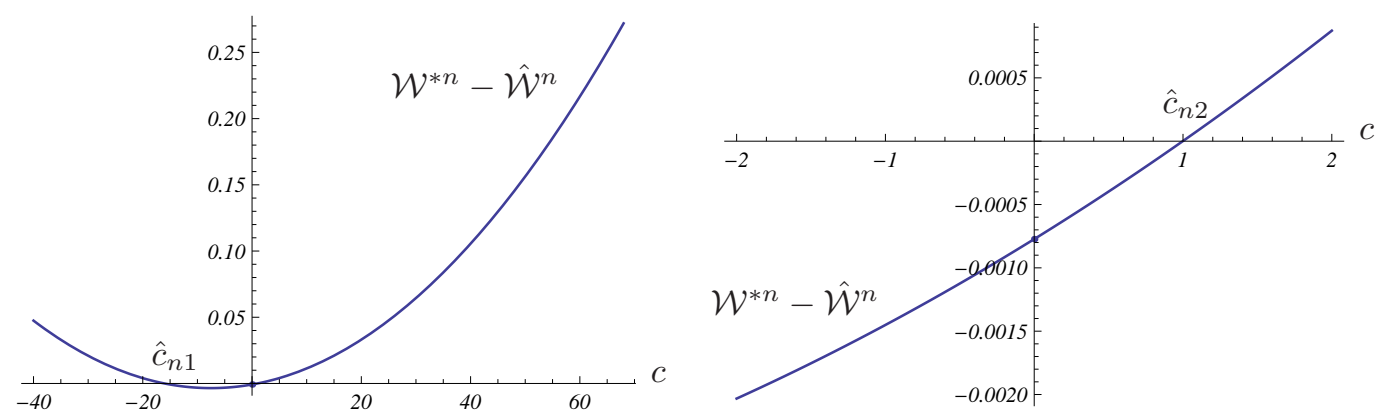

Figure 4: Welfare difference function $\hat{\mathcal{D}}^{n}$ in Sample $3(\xi=5,000)$

In Figure 3 we find $\hat{c}_{m 1}=-0.99$ and $\hat{c}_{m 2}=19.10$ satisfying $\hat{\mathcal{D}}^{m}\left(\hat{c}_{m 1}\right)=\hat{\mathcal{D}}^{m}\left(\hat{c}_{m 2}\right)=0$, $\hat{\mathcal{D}}_{c}^{m}\left(\hat{c}_{m 1}\right)<0$ and $\hat{\mathcal{D}}_{c}^{m}\left(\hat{c}_{m 2}\right)>0$. In Figure 4 there exist $\hat{c}_{n 1}=-16.03$ and $\hat{c}_{n 2}=0.99$ satisfying $\hat{\mathcal{D}}^{n}\left(\hat{c}_{n 1}\right)=\hat{\mathcal{D}}^{n}\left(\hat{c}_{n 2}\right)=0, \hat{\mathcal{D}}_{c}^{n}\left(\hat{c}_{n 1}\right)<0$ and $\hat{\mathcal{D}}_{c}^{n}\left(\hat{c}_{n 2}\right)>0$. Since $c_{\text {min }}=-40$ and $c_{\max }=68$, the ranking is ${ }^{19} c_{\min }<\hat{c}_{n 1}<\hat{c}_{m 1}<0<\hat{c}_{n 2}<\hat{c}_{m 2}<c_{\max }$. Table 7 illustrates the

\footnotetext{
${ }^{19}$ For countries belonging to the groups $M$ and $N$ the results are not mirror-symmetric, because the preferences for fuel of a free-riding $m$-country are invariant in $c$, while the preference parameter $a_{n}=100+c$ of a free-riding $n$-country obviously depends on $c$.
} 
conclusion regarding the stability of the grand coalition.

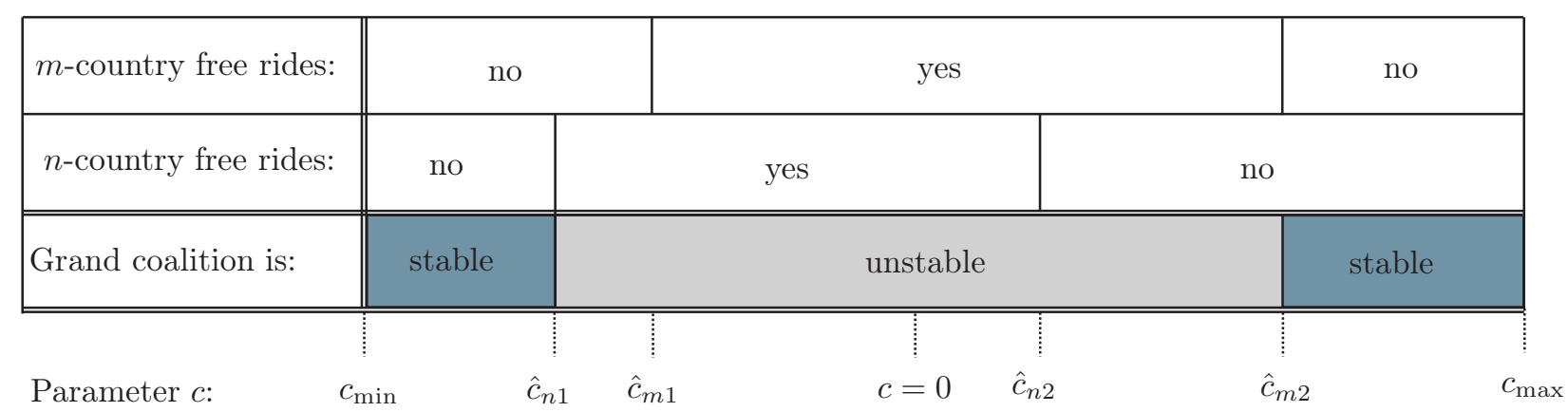

Table 7: Embargo and stability of the grand coalition in Sample $3(\xi=5,000)$

According to Table 7 the grand coalition is unstable in Sample 3 in the interval $] \hat{c}_{n 1}, \hat{c}_{m 2}[$ of zero, low and medium fuel-demand asymmetry $|c|$ and eventually becomes stable when $|c|$ is sufficiently large. This clearly suggests that asymmetric fuel demand improves the incentives to cooperate in the embargo regime, because increasing asymmetry raises the gains from trade among coalition countries, which in turn reduce the advantage of the autarkic free-riding country.

\begin{tabular}{|l||c|c|c|c|c|}
\hline Embargo regime & \multicolumn{2}{|c|}{ stable } & unstable & \multicolumn{2}{c|}{ stable } \\
\hline \hline Sub-interval no.: & 1 & 2 & 3 & 4 & 5 \\
\hline \hline Free-trade regime & stable & & unstable & stable \\
\hline \hline
\end{tabular}

Table 8: Stability of the grand coalition in the regimes of free trade and embargo in Sample $3(\xi=5,000)$

The next step towards examining the credibility of the threat of embargo in Sample 3 is to compare the intervals of stability and instability of the grand coalition in the regimes of free trade and embargo. The first row of Table 8 reproduces the last row of Table 7 , and the third row contains the outcome of Sample 3 in the free-trade regime "imported" from Eichner and Pethig (2014b, Table 5). ${ }^{20}$ The common feature of both regimes is that instability prevails in cases of zero and small to moderate asymmetry. If the asymmetry $|c|$ gets sufficiently large, the grand coalition stabilizes in both regimes, but the switch occurs at smaller degrees of asymmetry under embargo than under free trade. Although the embargo does not succeed in turning the grand coalition from instable to stable in all areas

\footnotetext{
${ }^{20}$ See also footnote 16.
} 
of asymmetry, in Sample 3 the embargo has the expected impact, to some extent at least, in contrast to the embargo in the Samples 1 and 2 above. More specifically, Table 8 shows that an embargo is ineffective at low levels of asymmetry (sub-interval 3; box [4] in Table 1), is unnecessary at very high levels of asymmetry (sub-intervals 1 and 5; box [1] of Table 1), and is effective at intermediate levels of asymmetry (sub-intervals 2 and 4; box [2] of Table 1 ). It is clear that the credibility of threatening to embargo free riders is not an issue in the economies belonging to the sub-intervals 1, 3 and 5, because in those economies an embargo makes no sense in the first place. The sub-intervals 2 and 4 are most interesting, because in these cases the embargo will do the job it is expected to do, if the threat of embargo turns out to be credible.

To examine the credibility in those sub-intervals, we recall from Table 2 the notation $\hat{w}_{h f}=\hat{\mathcal{W}}^{h f}(c)$ and $\bar{w}_{h f}=\overline{\mathcal{W}}^{h f}(c)$, according to which an $h f$-country (with $h, f=m, n$ ) is a country of group $H=M, N$ inside the coalition that is of size $m+n-1$ and faces an $f$-country, $f=m, n$, outside the coalition. Applying that notation, the threat of embargo is said to be credible, if

(i) $\hat{w}_{m m} \geq \bar{w}_{m m}$ and $\hat{w}_{n m} \geq \bar{w}_{n m}$ and

(ii) $\hat{w}_{m n} \geq \bar{w}_{m n}$ and $\hat{w}_{n n} \geq \bar{w}_{n n}$.

Since credibility requires satisfying conditions (i) and (ii), the threat of embargo fails to be credible if either the conditions (i) or the conditions (ii) or both conditions fail to hold.

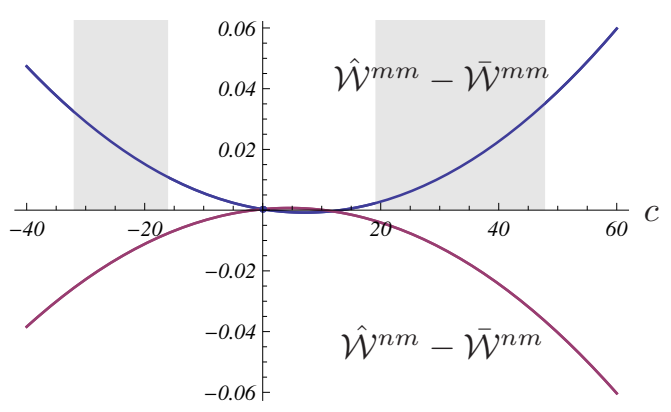

Figure 5: Credibility check in Sample $3(\xi=5,000)$

Figure 5 depicts the welfare differences $\hat{\mathcal{W}}^{h m}(c)-\overline{\mathcal{W}}^{h m}(c)$ on $\left[c_{\min }, c_{\max }\right]$ for $h=$ $m, n$. The shaded areas in that figure mark the intervals $\left[\bar{c}_{n 1}, \hat{c}_{n 1}\right]=[-32.36,-16.03]$ and $\left[\hat{c}_{m 2}, \bar{c}_{m 2}\right]=[19.10,47.86]$ on the $c$-axis that correspond to the sub-intervals 2 and 4 of Table 8 in which the transition from free trade to embargo stabilizes the grand coalition. A necessary condition for credibility is that $\hat{\mathcal{W}}^{m m}(c) \geq \overline{\mathcal{W}}^{m m}(c)$ and $\hat{\mathcal{W}}^{n m}(c) \geq \overline{\mathcal{W}}^{n m}(c)$ for 
some $c \in\left[\bar{c}_{n 1}, \hat{c}_{n 1}\right] \cup\left[\hat{c}_{m 2}, \bar{c}_{m 2}\right]$. Figure 5 shows, however, that this condition is not satisfied for any $c \in\left[\bar{c}_{n 1}, \hat{c}_{n 1}\right] \cup\left[\hat{c}_{m 2}, \bar{c}_{m 2}\right]$. Hence in all economies of Sample 3 in which an embargo could be useful it fails to be credible.

In Sample 3 the parameter $a$ is fixed at the level $a=100$. We wish to generalize the insight of Sample 3 - in a modest way - by investigating how the outcome of an embargo and its credibility change when we consider continuous variations of the parameters $c$ and $a$ while keeping unchanged all other parameters of Sample 3. To that end, we first consider the embargo regime. It can be shown that the points $c_{\text {min }}, \hat{c}_{n 1}$ and $\hat{c}_{m 1}$ are linearly decreasing in $a$ and $\hat{c}_{n 2}, \hat{c}_{m 2}$ and $c_{\max }$ are linearly increasing in $a$. The implication for the (in)stability of the grand coalition in the $(a, c)$ space is readily illustrated in Figure 6. The vertical line in Figure 6 illustrates the intervals of stability and instability of Sample 3 known from Table 7. Essentially, increasing the parameter $a$ amounts to linear expansions of the intervals of asymmetry in which the grand coalition is stable or instable leaving the order of these intervals unchanged. ${ }^{21}$ For any given value of $a$, the impact of variations of $c$ is as shown in Table 7 with the qualification that the asymmetry $|c|$ necessary to turn instability into stability is increasing in the parameter $a$. If we start with a tuple $(a, c)$ for which the grand coalition is stable and increase $a$ while keeping $c$ constant, we find some value $\tilde{a}$ such that the grand coalition is unstable for all feasible $\left(a^{\prime}, c\right)$ satisfying $a^{\prime}>\tilde{a}$.

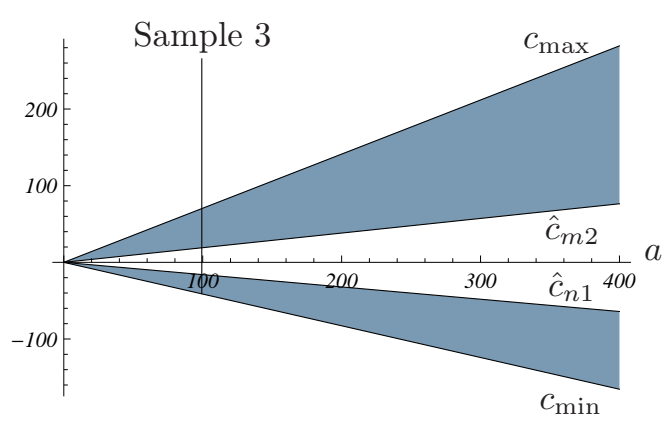

Figure 6: Stability of the grand coalition in the $(a, c)$ parameter space, when all other parameters are as in Sample $3(\xi=5,000)$

Eichner and Pethig (2014b, Figure 7) derive a figure similar to Figure 6 for Sample 3 in the free-trade regime. Rather than reproducing that figure here, we integrate Figure 7 from Eichner and Pethig (2014b) and Figure 6 of the present paper to obtain Figure 7 . In that figure, the cones shaded in grey give us the intervals $\left[\bar{c}_{n 1}(a), \hat{c}_{n 1}(a)\right]$ and $\left[\hat{c}_{m 2}(a), \bar{c}_{m 2}(a)\right]$ for

\footnotetext{
${ }^{21}$ This is also true for the Samples 4 and 5 we study below which is why we will not go through the exercise carried out in Figure 6 again in the subsequent analysis.
} 
all parameters $a$ for which an embargo is effective. The small stripe in red around the $a$-axis is the area of credibility. Since that stripe does not overlap with the grey cones anywhere the conclusion is

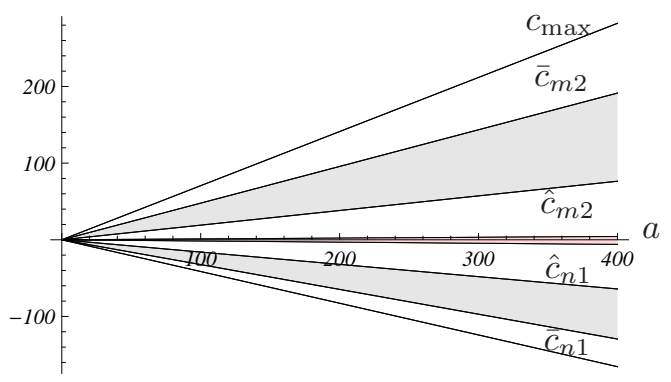

Figure 7: Check of credibility in the $(a, c)$-space, when all other parameters are as in Sample $3(\xi=5,000)$

Result 2. (Embargo and the size and asymmetry of fuel demand) Set the parameters $b=2,000, \delta=3, m=n=5, \rho=0$ and $\xi=5,000$ as in Sample 3 and consider all feasible pairs $(a, c)$. Threatening to embargo free riders is not suitable as a policy to forge the grand climate coalition for any pair $(a, c)$, because the embargo is either ineffective or unnecessary or it is effective but not credible.

\subsection{Size and asymmetry of fuel demand and size of extraction costs}

The Sample 3 we discussed above is characterized by medium-level extraction costs. Now we turn to the cases of lower (Sample 4) and higher (Sample 5) extraction costs.
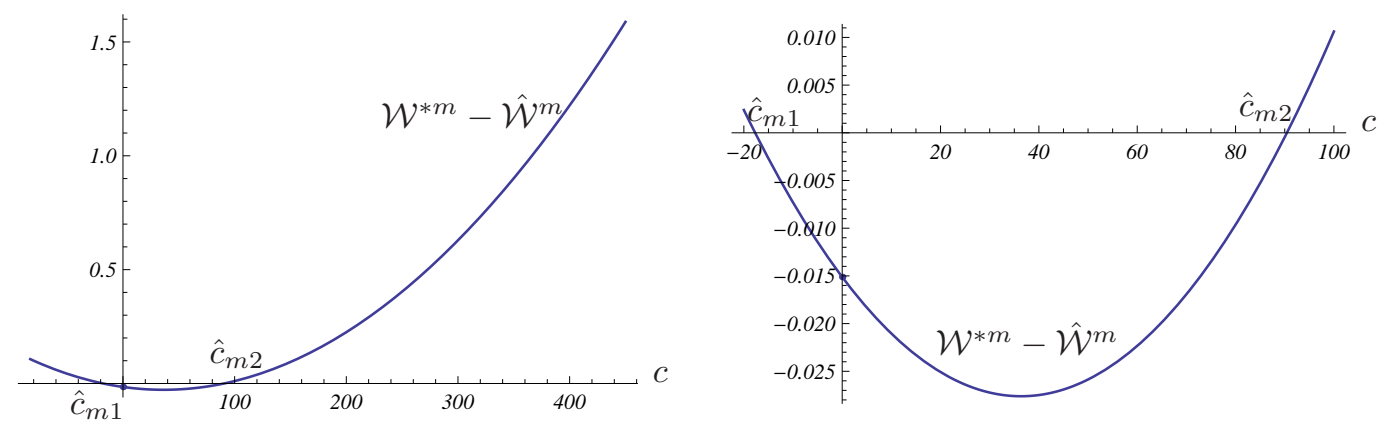

Figure 8: Welfare difference function $\hat{\mathcal{D}}^{m}$ in Sample $4(\xi=500)$ 

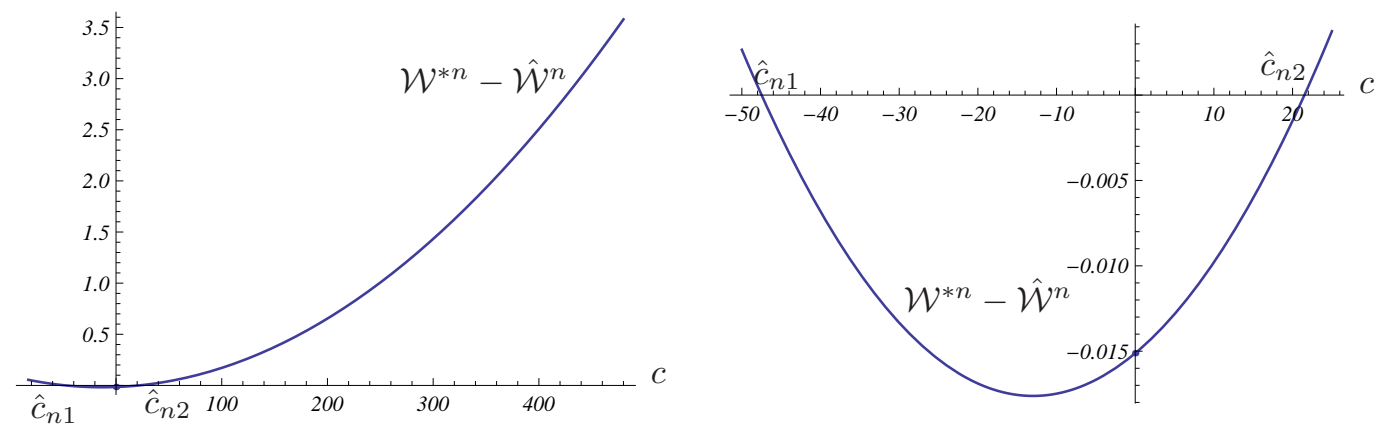

Figure 9: Welfare difference function $\hat{\mathcal{D}}^{n}$ in Sample $4(\xi=500)$

The Figures 8 and 9 correspond to Sample 4. In Figure 8 we find $\hat{c}_{m 1}=-17.70$ and $\hat{c}_{m 2}=90.45$ satisfying $\hat{\mathcal{D}}^{m}\left(\hat{c}_{m 1}\right)=\hat{\mathcal{D}}^{m}\left(\hat{c}_{m 2}\right)=0, \hat{\mathcal{D}}_{c}^{m}\left(\hat{c}_{m 1}\right)<0$ and $\hat{\mathcal{D}}_{c}^{m}\left(\hat{c}_{m 2}\right)>0$. In Figure 9 there exist $\hat{c}_{n 1}=-47.5$ and $\hat{c}_{n 2}=21.51$ satisfying $\hat{\mathcal{D}}^{n}\left(\hat{c}_{n 1}\right)=\hat{\mathcal{D}}^{n}\left(\hat{c}_{n 2}\right)=0, \hat{\mathcal{D}}_{c}^{n}\left(\hat{c}_{n 1}\right)<0$ and $\hat{\mathcal{D}}_{c}^{n}\left(\hat{c}_{n 2}\right)>0$. Since $c_{\min }=-83$ and $c_{\max }=479$, the ranking is ${ }^{22} c_{\min }<\hat{c}_{n 1}<\hat{c}_{m 1}<$ $0<\hat{c}_{n 2}<\hat{c}_{m 2}<c_{\max }$. We conclude that in Sample 4 the pattern of stability and instability of the grand coalition is the same as in Table 7 for Sample 3, which is why there is no need to reproduce that table here for Sample 4. An embargo succeeds to stabilize the grand coalition, if the fuel-demand asymmetry is sufficiently large.

\begin{tabular}{|c|c|c|c|}
\hline Embargo regime: & stable & unstable & stable \\
\hline Sub-interval no.: & 1 & 2 & 3 \\
\hline Free-trade regime: & \multicolumn{3}{|c|}{ unstable } \\
\hline
\end{tabular}

Table 9: Embargo and stability of the grand coalition in Sample $4(\xi=500)$

The first row of Table 9 shows the stability pattern of Sample 4 in the embargo regime, and the third row of this table, taken from Eichner and Pethig (2014b, Table 7), describes the outcome of Sample 4 in the free-trade regime. According to Table 9 an embargo is either

\footnotetext{
${ }^{22}$ The results for $m$ - and $n$-countries are not mirror-symmetric, because the preferences for fuel of a freeriding $m$-country are invariant in $c$, while the preference parameter $a_{n}=100+c$ of a free-riding n-country obviously depends on $c$.
} 
ineffective (sub-interval 2) or effective (sub-intervals 1 and 3) which requires examining the credibility of the threat of embargo in economies belonging to the sub-intervals 1 and 3 .
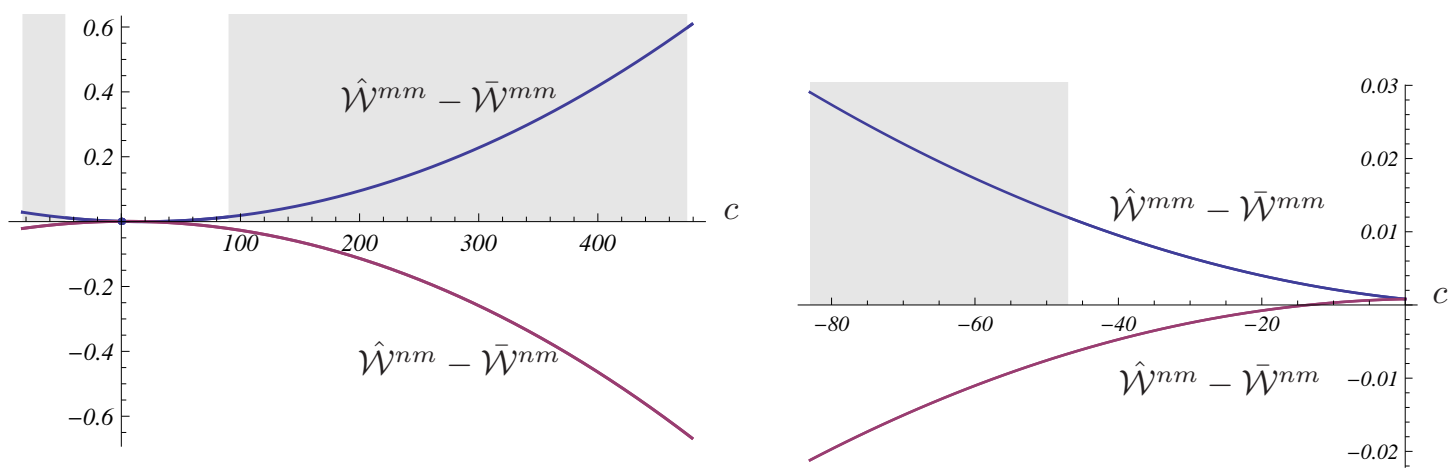

Figure 10: Credibility check in Sample $4(\xi=500)$

Figure 10 depicts the welfare differences $\hat{\mathcal{W}}^{h m}(c)-\overline{\mathcal{W}}^{h m}(c)$ on $\left[c_{\min }, c_{\max }\right]$ for $h=m, n$. The shaded area in the left and right panel of Figure 10 marks the sub-interval 4 and 2 of Table 8, respectively, in which the transition from free trade to embargo stabilizes the grand coalition. We conclude that there is no $|c|$ in the shaded intervals for which both curves are positive valued. Hence in all economies of Sample 4 in which an embargo could be useful it fails to be credible.

Finally, we turn to Sample 5 that represents the case of high extraction costs, much higher than in the Samples 3 and 4. The welfare difference curves of Sample 5 are plotted in Figures 11 and 12. In Figure 11 we find $\hat{c}_{m 1}=-0.004$ and $\hat{c}_{m 2}=1.18$ satisfying $\hat{\mathcal{D}}^{m}\left(\hat{c}_{m 1}\right)=$ $\hat{\mathcal{D}}^{m}\left(\hat{c}_{m 2}\right)=0, \hat{\mathcal{D}}_{c}^{m}\left(\hat{c}_{m 1}\right)<0$ and $\hat{\mathcal{D}}_{c}^{m}\left(\hat{c}_{m 2}\right)<0$. In Figure 12 there exist $\hat{c}_{n 1}=-1.17$ and $\hat{c}_{n 2}=0.004$ satisfying $\hat{\mathcal{D}}^{n}\left(\hat{c}_{n 1}\right)=\hat{\mathcal{D}}^{n}\left(\hat{c}_{n 2}\right)=0, \hat{\mathcal{D}}_{c}^{n}\left(\hat{c}_{n 1}\right)<0$ and $\hat{\mathcal{D}}_{c}^{n}\left(\hat{c}_{n 2}\right)>0$. Since $c_{\min }=-3.5$ and $c_{\max }=3.6$, the ranking is $c_{\min }<\hat{c}_{n 1}<\hat{c}_{m 1}<0<\hat{c}_{n 2}<\hat{c}_{m 2}<c_{\max }$.

The Figures 11 and 12 show that in Sample 5 the pattern of stability and instability of the grand coalition is the same as in Table 7 for Sample 3, which is why we need not reproduce that table for Sample 5. Interestingly, in the embargo regime it is the common feature of the Samples 3, 4 and 5 that in case of sufficiently large asymmetry the grand coalition turns from instable to stable. This pattern does not hold in the free-trade regime, 

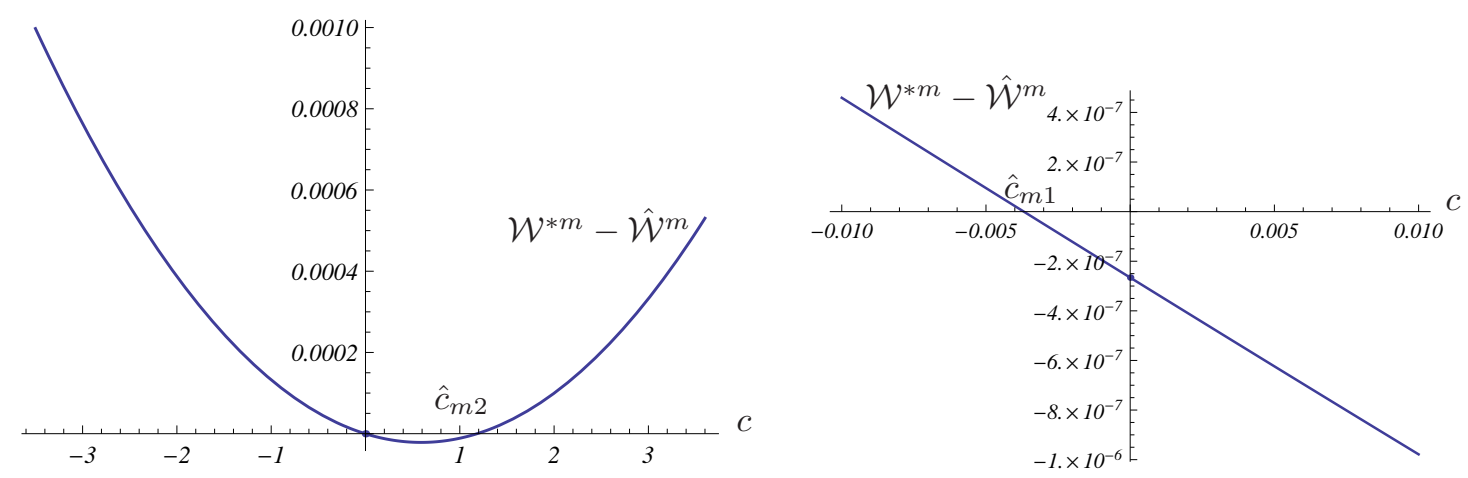

Figure 11: Welfare difference function $\hat{\mathcal{D}}^{m}$ in Sample $5(\xi=100,000)$
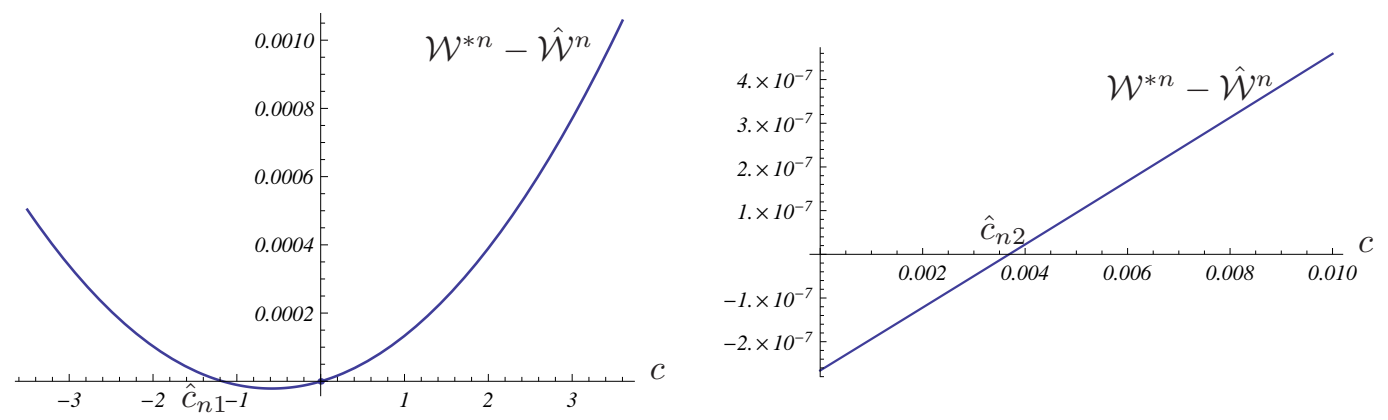

Figure 12: Welfare difference function $\hat{\mathcal{D}}^{n}$ in Sample $5(\xi=100,000)$

however, as the comparison of the last rows of the Tables 8, 9 and 10 shows. $^{23}$ In the free-trade regime, the common feature of the Samples 2 and 5 is that the grand coalition is stable in cases of symmetry and small asymmetries $(|\rho| \neq 0$ in Sample 2 and $|c| \neq 0$ in Sample 5), because a low damage parameter $\delta$ as well as a high extraction cost parameter $\rho$ keep climate damage low. The Samples 2 and 5 differ (in the free-trade regime), however, because large asymmetries restore stability in Sample 5 but not in Sample 2. Observe also that under free trade, the effect of asymmetry on stability is "non-linear" in Sample 5. The grand coalition is stable in case of full symmetry, it gets unstable at intermediate levels of fuel-demand asymmetry, and it stabilizes again when the asymmetry becomes sufficiently large.

Inspection of Table 10 reveals that the set of feasible asymmetries $\left[c_{\min }, c_{\max }\right]$ is partitioned into seven sub-intervals that cover all possible combinations, listed in Table 1, between stability and instability in the transition from free trade to embargo. ${ }^{24}$ As above, we restrict

\footnotetext{
${ }^{23}$ The last row in Table 10 is taken from Table 8 in Eichner and Pethig (2014b).

${ }^{24}$ The explanation of the feature that an embargo destabilizes the otherwise stable grand coalition in the
} 


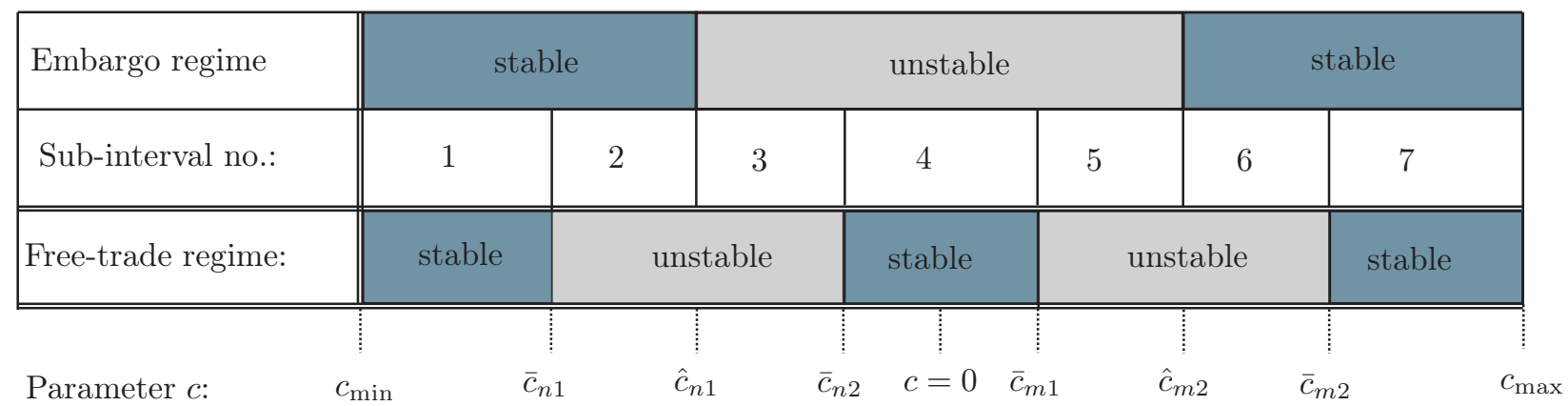

Table 10: Stability of the grand coalition in the regimes of free trade and embargo in Sample 5

$$
(\xi=100,000)
$$

our attention to the sub-intervals in which an embargo stabilizes the otherwise unstable grand coalition, if the threat of embargo turns out to be credible.

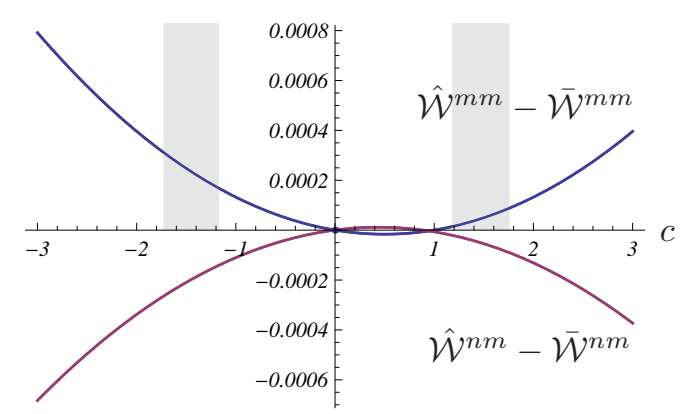

Figure 13: Credibility check in Sample $5(\xi=100,000)$

Figure 13 depicts the welfare differences $\hat{\mathcal{W}}^{h m}(c)-\overline{\mathcal{W}}^{h m}(c)$ on $\left[c_{\min }, c_{\max }\right]$ for $h=m, n$. The shaded areas in Figure 13 mark the sub-intervals 2 and 6 of Table 10, in which the transition from free trade to embargo stabilizes the grand coalition. According to Figure 13, there is no $|c|$ in the shaded intervals for which both curves are positive valued. Hence in all economies of Sample 5 in which an embargo could be useful it fails to be credible.

In the preceding analysis, we showed for three different values of the extraction cost parameter $\xi$ that there is no subset of parameters in the Samples 3, 4 and 5, for which the threat of embargo is credible in cases where the imposition of the embargo would stabilize the otherwise unstable grand coalition. In the sequel, we generalize by showing that this result holds not only for the values $\xi=500, \xi=5,000$ and $\xi=100,000$ of the Samples 4, 3 and 5 , respectively, but also for all positive $\xi$. Specifically, we will characterize the parameter sub-interval 4 of Table 10 is the same as in Sample 2 (sub-interval 2 of Table 5). 
space $(c, \xi)$ for given parameters $\delta=3, a=100, m=n=5, b=2,000$ and $\rho=0$ which are common to the Samples 3 - 5. Figure 14 illustrates the result of that exercise for the embargo regime. The areas in Figure 14 shaded in blue mark those subsets of parameters $(c, \xi)$ in which the grand coalition is stable in the embargo regime. For the values of $\xi$ characterizing the Samples 3, 4 and 5, Figure 14 illustrates the first rows of the Tables 8, 9 and 10 from a different perspective. Moreover, Figure 14 shows that there is $\hat{\xi}=41.7$ such that this pattern of stability and instability in the Samples $3-5$ holds for all $\xi>\hat{\xi}$, whereas the grand coalition is instable on $\left[c_{\min }(\xi), c_{\max }(\xi)\right]$ for all $\xi \leq \hat{\xi}$.

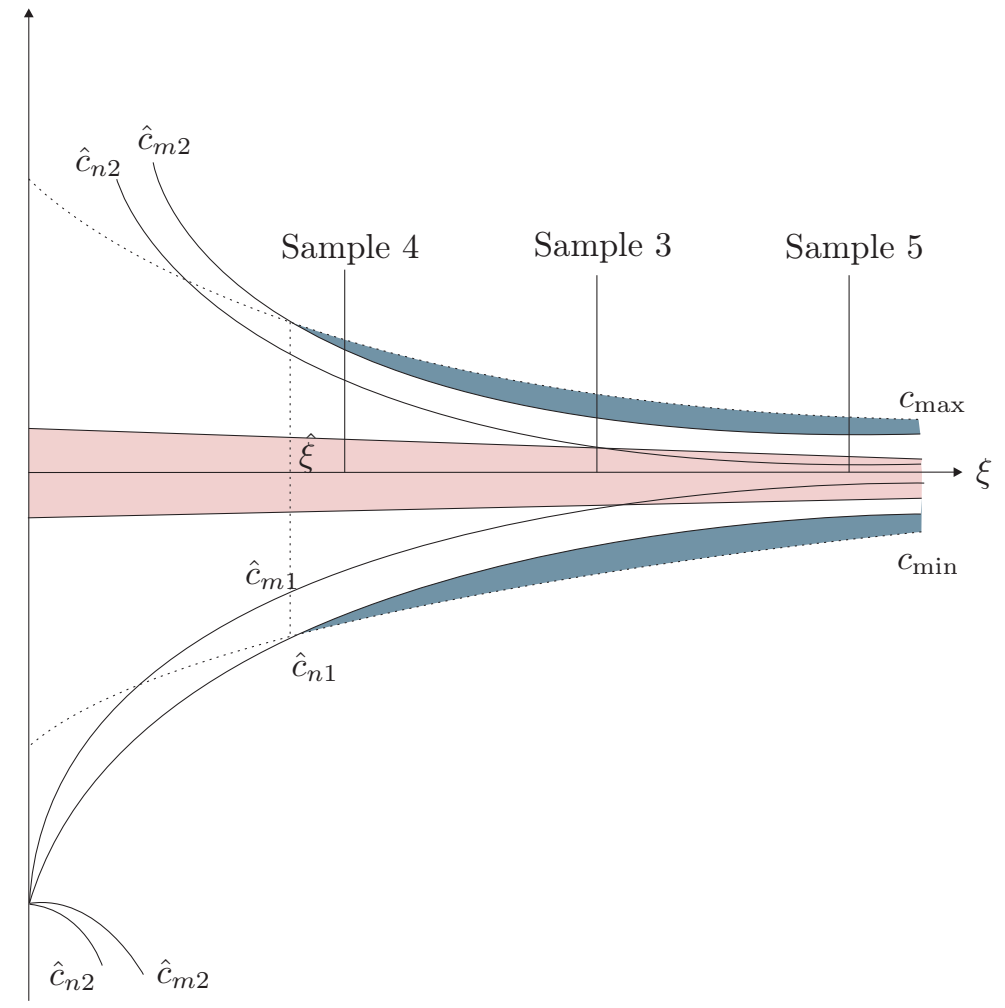

Figure 14: Embargo and stability of the grand coalition in the $(c, \xi)$ parameter space

Figure 14 also answers the question whether the threat of embargo is credible in scenarios where the transition from free trade to embargo stabilizes the grand coalition. The area in Figure 14 shaded in red is the set of all pairs $(a, c)$ for which the countries of both groups $M$ and $N$ in the coalition of size $m+n-1$ are at least as well off in the embargo regime as in the free-trade regime. A necessary condition for the credibility of the threat of embargo in cases where the embargo stabilizes the otherwise unstable grand coalition is that the red area and the blue area overlap. The absence of such an overlap in Figure 14 proves that in all economies covered by Figure 14 - and hence a fortiori in all economies of the Samples 3, 4 and 5 - in which an embargo stabilizes the grand coalition the threat of embargo fails to be credible. The insights of Section 5.2 are summarized in 
Result 3. (Embargo, size and asymmetry of fuel demand, and size of extraction costs) Set the parameters $a=100, b=2000, \delta=3, m=n=5$ and $\rho=0$ that are common to the Samples 3, 4 and 5 and consider all feasible pairs $(c, \xi)$. Threatening to embargo free riders is not suitable as a policy to forge the grand climate coalition for any pair $(c, \xi)$, because the embargo is either ineffective or counterproductive or unnecessary or it is effective but not credible.

\section{Credibility via intra-coalition transfers?}

In this section, we investigate whether transfers within the coalition can solve the credibility problem. We now introduce the assumption that if the aggregate welfare of the coalition is at least as large in the embargo, $\hat{\mathcal{W}}^{z f}$, as in the free-trade regime, $\overline{\mathcal{W}}^{z f}$, the coalition countries conduct intra-coalition transfers that make all coalition countries at least as well off in the embargo as in the free-trade regime. The $f$-country is supposed to anticipate the coalition countries' commitment to such intra-coalition transfers and hence considers credible the threat of embargo, if $\hat{\mathcal{W}}^{z f} \geq \overline{\mathcal{W}}^{z f}$. In Figure 15, we examine whether the credibility can be regained in the Samples 3 and 5 in those cases in which the transition from free trade to embargo was shown to stabilize the grand coalition. In both panels of Figure 15, the u-shaped curves are the aggregate welfare differences $\hat{\mathcal{W}}^{z f}(c)-\overline{\mathcal{W}}^{z f}(c)$ for $f=m$ and the inverse $\mathrm{u}$-shaped curves are those welfare differences for $f=n$. The welfare differences in both curves must be positive in order to make the threat of embargo credible via transfers. The grey areas in Figure 15 mark the sub-intervals in which the embargo stabilizes the otherwise unstable grand coalition. In the left panel of Figure 15, that illustrates Sample 5, no parameter $c$ exists in the grey areas for which both welfare differences are positive. Hence, transfers fail to restore credibility in Sample 5. However, the result is different Sample 3 in the right panel of Figure 15. In the grey area of that panel, we find an interval in which both welfare differences are positive. In that interval, the threat of embargo is credible, if combined with appropriate transfers.

The results of Samples 3 and 5 are generalized in the Figures 16 and 17. In the red areas of these figures, the embargo is credible, and in the grey areas the grand coalition is unstable under free trade but stable in the embargo regime. Figure 16 and the left panel of Figure 17 show that the red and grey areas do not overlap. It follows that for all $\xi>\breve{\xi}=17,203$ the threat of embargo with transfers is not credible. In contrast, if $\xi \in[\hat{\xi}, \breve{\xi}]$ there is an overlap of the grey and red areas which is colored in blue in the right panel of Figure 17. We summarize the findings of Figures 16 and 17 in 


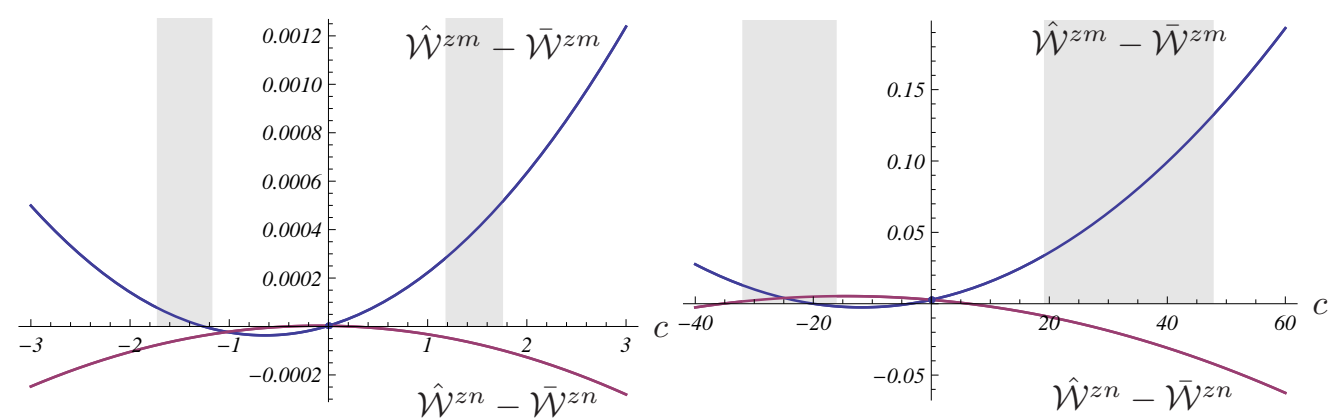

Figure 15: Credibility with transfers in Sample $5(\xi=100,000$, left panel $)$ and in Sample 3 $(\xi=5,000$, right panel $)$

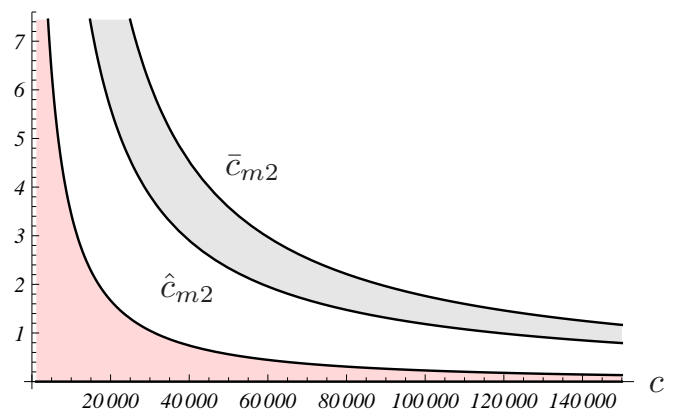

Figure 16: Credibility with transfers in the $(c, \xi)$ parameter space for positive $c$ 's
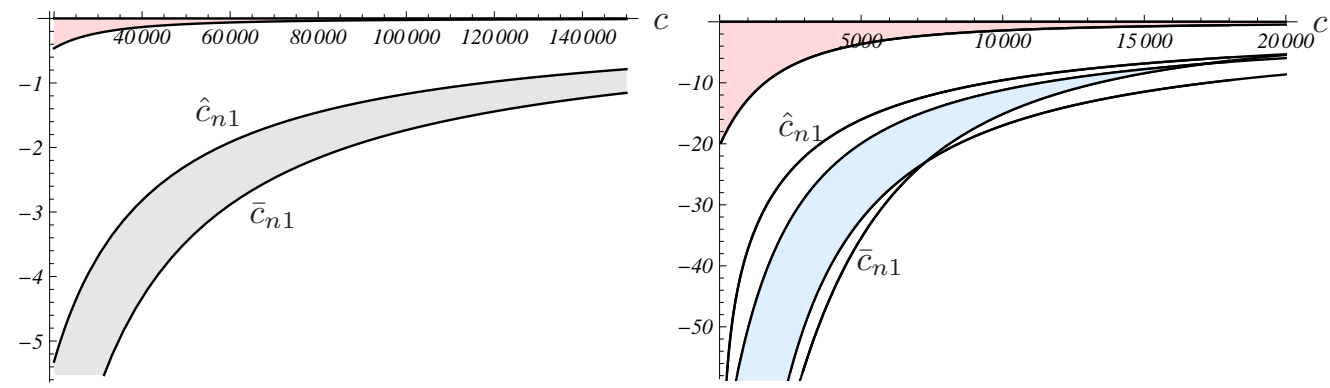

Figure 17: Credibility with transfers in the $(c, \xi)$ parameter space for negative $c$ 's

Result 4. (Embargo with transfers, size and asymmetry of fuel demand, and size of extrac- 
tion costs)

Consider the parameters $a=100, b=2,000, \delta=3, m=n=5$ and $\rho=0, \hat{\xi}=41.7$ and $\breve{\xi}=17,203$ that are common to the Samples 3, 4 and 5 and consider all feasible pairs (c, $\xi)$. There exist $\hat{\xi}$ and $\breve{\xi}, 0<\hat{\xi}<\breve{\xi}$, such that the following holds.

(i) If the extraction costs are sufficiently low $(\xi<\hat{\xi})$ or sufficiently high $(\xi>\breve{\xi})$, the threat of embargo with transfers is not suitable as a policy to forge the grand climate coalition for any pair $(c, \xi)$, because the embargo is either ineffective or counterproductive or unnecessary or it is effective but not credible.

(ii) If the extraction costs are intermediate $\xi \in[\hat{\xi}, \breve{\xi}]$, there exists a sub-interval $\mathcal{C} \subset\left[\bar{c}_{n 1}, \hat{c}_{n 1}\right]$ such that the threat of embargo with transfers is suitable as a policy to forge the grand climate coalition for any $c \in \mathcal{C}$, because the embargo is both effective and credible.

\section{Concluding remarks}

The paper analyzes the suitability of threatening to embargo free riders as an instrument to forge a global self-enforcing IEA - or a stable grand coalition. It is based on the model of Eichner and Pethig (2014b) that features production and consumption of fossil fuel and a final good, international trade in these goods, carbon taxes and two groups of countries that differ with respect to climate damage and fossil fuel demand. It extends that model by investigating whether the threat of embargo can induce countries to sign a global IEA. Despite very simple parametric modeling, the calculations to answer that question are so complex that informative general results cannot be obtained. We therefore proceed with simulations in the form of five samples, each of which contains continuous changes of one of the asymmetry parameters. Our results do not support the expectation - or hope - that the threat of embargo is a forceful lever to foster global climate cooperation.

The first two samples focus on climate damage asymmetries. For any degree of asymmetry, we find that if the grand coalition is unstable under free trade, it does not turn stable in the embargo regime, and if the grand coalition is stable under free trade, an embargo destabilizes it. Hence, an embargo is either useless or even counterproductive in these samples suggesting that asymmetric climate damage weakens the incentives to cooperate. Clearly, the credibility of the threat of embargo is not an issue in the first two samples, because here the threat does not make sense in the first place. The other three samples investigate continuous variations of the degree of fuel-demand asymmetry for three different levels of extraction costs. Overall, our simulations suggest that increasing degrees of fuel-demand asymmetry tend to encourage the incentives to cooperate in the transition from free trade 
to the embargo regime. Specifically, for low extraction costs (Sample 4) the grand coalition is unstable in the free-trade regime and remains unstable with embargo. In case of higher extraction costs (Samples 3 and 5) the grand coalition may be stable in the embargo regime and there are (small) subsets of parameters, referred to as the set $Z$ of world economies, in which the grand coalition is unstable under free trade, but stable in the embargo regime. That is, in world economies in the set $Z$, the embargo accomplishes what one may have hoped it would always do.

However, that result is good news only after showing that the threat of embargo is credible the world economies belonging to the set $Z$. Unfortunately, we find that the threat is not credible for any world economy in $Z$, no matter how low or high the degree of asymmetry is. Consequently, the threat of embargo is not suitable as an instrument to forge the grand coalition in any simulation we conducted, because it is either ineffective or counterproductive or it is effective but not credible. Given this negative result, we briefly turn back to the world economies in the set $Z$ and examine whether it is possible to gain credibility through intra-coalition transfers that make all coalition countries better off under embargo than under free trade. We find a glimmer of hope, because such transfers establish credibility, in fact, on a small subset of the set $Z$.

\section{References}

Barrett, S. (2003): Environment and Statecraft. The Strategy of Environmental Treatymaking, Oxford University Press, Oxford.

Barrett, S. (2001): International cooperation for sale. European Economic Review 45(10), 1835-1850.

Barrett, S. (1999): The credibility of trade sanctions in international environmental agreements, Per Fredriksson (ed.), Trade, Global Policy, and the Environment, 161-.

Barrett, S. (1997): The strategy of trade sanctions in international environmental agreements, Resource and Energy Economics 19, 345-361.

Barrett, S. (1994): Self-enforcing international environmental agreements. Oxford Economic Papers 46, 878 - 894.

Brack, D. (1996): International Trade and the Montreal Protocol, Royal Institute of International Affairs, London.

Carraro, C. and D. Siniscalco (1998): International Environmental agreements: incentives 
and political economy. European Economic Review 42, 561-572.

Charnovitz, S. (2007): The WTO's environmental progress, Journal of International Economic Law 10, 685-706.

Chui Ying, Martina Lui (2010): Are Economic Sanctions Credible and Effective In Deterring Free-Riding of an International Environmental Agreement? Manuscript, BBA Business Economics (Hons), City University of Hong Kong (chuiying@uvic.ca).

D'Aspremont, L., Jacquemin, A., Gabszewicz, J.J and J.A. Weymark (1983): On the stability of collusive price leadership. The Canadian Journal of Economics 16, 17-25.

Diamantoudi, E. and E. Sartzetakis (2006): Stable international environmental agreements: An analytical approach. Journal of Public Economic Theory 8, 247-263.

Eichner, T. and R. Pethig (2014a): Self-enforcing environmental agreements and trade: taxes versus caps. CESifo Working Paper No. 4954.

Eichner, T. and R. Pethig (2014b): Global environmental agreements, international trade, and asymmetric countries. CESifo Working Paper No. 5051.

Eichner, T. and R. Pethig (2013): Self-enforcing environmental agreements and international trade. Journal of Public Economics 102, 37 - 50.

Elliot, J., Foster, I., Kortum, S., Munson, T., Perez Cervantes, F. and D. Weisbach (2010), Trade and carbon taxes. American Economic Review: Papers $\&$ Proceedings 1000, 465-469.

Finus, M. (2003): Stability and design of international environmental agreements: The case of transboundary pollution, in: H. Folmer and T. Tietenberg (eds.), The International Yearbook of Environmental and Resource Economics 2003/2004, Edward Elgar, Cheltenham, 82 - 158.

Fuentes-Abero, C. and S. Rubio (2010): Can international environmental cooperation be bought? European Journal of Operations Research 202, 255-264.

Kemfert, C. (2004): Climate coalitions and international trade: assessment of cooperation incentives by issue linkage, Energy Policy 32, 455-465.

Lessmann, K., R. Marschinski and O. Edenhofer (2009): The effects of tariffs on coalition formation in a dynamic global warming game, Economic Modelling 26, 641-649.

McGinty, M (2007): International environmental agreements among asymmetric nations. Oxford Economic Papers 59, 45-62. 
Pavlova, Y. and A. de Zeeuw (2013): Asymmetries in international environmental agreements. Environment and Development Economics 18, 51-68.

Rubio, S.J. and A. Ulph (2006): Self-enforcing agreements and international trade in greenhouse emission rights. Oxford Economic Papers 58, 233-263.

Stiglitz, J.E. (2006): Making Globalization Work. W.W. Norton \&6 Company, New York.

Van Slooten (1994): The case of the Montreal Protocol, in: OECD (ed.), Trade and Environment: Processes and Production Methods, Paris, 87-90.

\section{Appendix}

\section{A: Derivation of the Nash equilibrium tax rates $\hat{t}_{f}$ and $\hat{t}_{z f}$}

The free-riding country maximizes $\hat{W}^{f}\left(t_{f}, t_{z}, c, \rho\right)$ from (16) with respect to $t_{f}$. The associated first-order condition reads

$$
\hat{W}_{t_{f}}^{f}= \begin{cases}\frac{\partial e_{f}^{d}}{\partial t_{f}} t_{f}-\delta\left[e_{f}^{s}+(m+n-1) e_{z f}^{s}\right] \frac{\partial e_{f}^{s}}{\partial t_{f}}=0 & \text { if } f=m, \\ \frac{\partial e_{f}^{d}}{\partial t_{f}} t_{f}-(\delta+\rho)\left[e_{f}^{s}+(m+n-1) e_{z f}^{s}\right] \frac{\partial e_{f}^{s}}{\partial t_{f}}=0 & \text { if } f=n .\end{cases}
$$

The coalition maximizes $\hat{W}^{z f}\left(t_{f}, t_{z}, c, \rho\right)$ from (15) with respect to $t_{z}$. The corresponding first-order condition is

$$
\hat{W}_{t_{z}}^{z f}= \begin{cases}(m-1) \frac{\partial e_{m f}^{d}}{\partial t_{z}} t_{z}+n \frac{\partial e_{n f}^{d}}{\partial t_{z}} t_{z} & \text { if } f=m \\ -(m+n-1) \delta\left[e_{f}^{s}+(m+n-1) e_{z f}^{s}\right] \frac{\partial e_{z f}^{s}}{\partial t_{z}}=0 & \\ m \frac{\partial e_{m f}^{d}}{\partial t_{z}} t_{z}+(n-1) \frac{\partial e_{n f}^{d}}{\partial t_{z}} t_{z} & \text { if } f=n . \\ -(m+n-1)(\delta+\rho)\left[e_{f}^{s}+(m+n-1) e_{z f}^{s}\right] \frac{\partial e_{z f}^{s}}{\partial t_{z}}=0\end{cases}
$$

Solving (A1)-(A2) yields

$$
\begin{aligned}
\hat{T}^{m}(c, 0) & =\hat{T}^{n}(c, 0)=\frac{c n+a(m+n) \delta}{\alpha+b\left[2+m^{2}+2 m(n-1)-2 n+n^{2}\right] \delta}, \\
\hat{T}^{z m}(c, 0) & =\hat{T}^{z n}(c, 0)=\frac{(n+m-1)[c n+a(m+n)]}{\alpha+b+\left[2+m^{2}+2 m(n-1)-2 n+n^{2}\right] \delta}, \\
\hat{T}^{m}(0, \rho) & =\frac{a(m+n) \delta}{\alpha+b+\left[2+m^{2}+2 m(n-1)-2 n+n^{2}\right] \delta+n(n+m-1) \rho} \\
\hat{T}^{z m}(0, \rho) & =\frac{a(m+n)[(n+m-1) \delta+n \rho]}{\alpha+b+\left[2+m^{2}+2 m(n-1)-2 n+n^{2}\right] \delta+n(n+m-1) \rho} \\
\hat{T}^{n}(0, \rho) & =\frac{a(m+n)(\delta+\rho)}{\alpha+b+\left[2+m^{2}+2 m(n-1)-2 n+n^{2}\right] \delta+\left(n^{2}-2 n+m n+2-m\right) \rho} \\
\hat{T}^{z n}(0, \rho) & =\frac{a(m+n)[(n+m-1) \delta+(n-1) \rho]}{\alpha+b+\left[2+m^{2}+2 m(n-1)-2 n+n^{2}\right] \delta+\left(n^{2}-2 n+m n+2-m\right) \rho} .
\end{aligned}
$$




\section{B: The domain of the functions $\hat{\mathcal{W}}^{f}: \mathcal{D} \rightarrow \mathbb{R}$ for $f=m, n$}

In the sequel we restrict the set of feasible parameters such that all fossil fuel demand and supply and all final good supplies and demands are strictly positive. In addition, we assume $m=n$. The set of feasible parameters for the functions $W^{* f}$ and $\bar{W}^{f}$ is derived in Eichner and Pethig (2014b).

Next, consider the functions $\hat{\mathcal{W}}^{f}$ and suppose that $c \neq 0$ and $\rho=0$. Inserting the Nash-equilibrium tax rates (A3) - (A4) in (13) - (14) for $\rho=0$ we get in the embargo equilibrium if country $m$ free rides:

$$
\begin{array}{rll}
e_{f}^{s}=e_{f}^{d}>0 & \Longleftrightarrow & c<\frac{a[\xi+b+2[n(2 n-3)+1] \delta]}{n \delta}=: \hat{c}_{1}, \\
e_{z m}^{s}>0 & \Longleftrightarrow \quad c>-\frac{a(2 n-1)[\xi+b-2(n-1) \delta]}{n(\xi+b+\delta)}=: \hat{c}_{2}, \\
e_{m m}^{d}>0 & \Longleftrightarrow \quad c<\frac{a b(2 n-1)[\xi+b-2(n-1) \delta]}{n \xi(\alpha+b)+\left[(\xi+b)(2 n-1)^{2}+\xi\right] n \delta}=: \hat{c}_{3}, \\
e_{n m}^{d}>0 & \Longleftrightarrow \quad c>-\frac{a b(2 n-1)[\xi+b-2(n-1) \delta]}{(\xi+b)[(2 n-1) b+(n-1) \xi]+[[(n-1)(2 n-1)+1] b+2[2 n(n-1)+1] \xi]] \delta}=: \hat{c}_{4} .
\end{array}
$$

If country $n$ free rides, the embargo equilibrium is characterized by

$$
\begin{aligned}
e_{f}^{s}=e_{f}^{d}>0 & \Longleftrightarrow c>-\frac{a[\xi+b+(2 n-1)(2 n-2) \delta]}{\xi+b+\left[2(n-1)^{2}+n(2 n-1)\right] \delta}=: \hat{c}_{5}, \\
e_{z n}^{s}>0 & \Longleftrightarrow c>-\frac{a(n+m-1)[\xi+b-2(n-1) \delta]}{\left.(n-1)(\xi+b)-\left[2(n-1)^{2}+n(2 n-1)\right] \delta\right)}=: \hat{c}_{6}, \\
e_{m n}^{d}>0 & \Longleftrightarrow c<\frac{a b(2 n-1)[\xi+b-2(n-1) \delta]}{\alpha(\xi+b)(n-1)^{2}+\left[n(2 n-1)^{2} b+(n-1)[2 n(2 n-1)-2(n-1)] \xi\right] \delta}=: \hat{c}_{7}, \\
e_{n n}^{d}>0 & \Longleftrightarrow c>-\frac{a b(2 n-1)[\xi+b-2(n-1) \delta]}{(\xi+b)[(2 n-1) b+n \xi]+\left[(2 n-1)[(n-1)(2 n-1)+1] b+n\left[2(n-1)^{2}+2 n^{2}\right] \xi\right] \delta}=: \hat{c}_{8} .
\end{aligned}
$$

Observe that $\hat{c}_{1}>0, \hat{c}_{5}<0$. In the sequel we assume $b+\frac{2 n-1}{2 n^{2}} \xi>2(n-1) \delta$ which is sufficient for $\hat{c}_{2}<0, \hat{c}_{3}>0, \hat{c}_{4}<0, \hat{c}_{6}<0, \hat{c}_{7}>0$, $\hat{c}_{8}<0$. Moreover, sufficiently large values of $\bar{x}$ ensure that all final good supplies and demands are strictly positive. It follows that for sufficiently 
large parameters $\bar{x}$ the domain of the functions $\mathcal{W}^{* h}(c, 0), \overline{\mathcal{W}}^{f}(c, 0)$ and $\hat{\mathcal{W}}^{f}(c, 0)$ is

$$
\mathcal{S}^{c}:=\left\{(a, b, \xi, n, \delta, c, \rho) \mid(a, b, \xi, n, \delta, c) \in \mathbb{R}_{++}^{6}, \rho=0, n \geq 2, b+\frac{2 n-1}{2 n^{2}} \xi>2(n-1) \delta \wedge c \in\left[c_{\text {min }}, c_{\text {max }}\right]\right\},
$$

where $^{25} c_{\min }:=\max \left\{c_{2}^{*}, c_{3}^{*},-a, \bar{c}_{1}, \bar{c}_{3}, \bar{c}_{5}, \bar{c}_{7}, \bar{c}_{8}, \hat{c}_{2}, \hat{c}_{4}, \hat{c}_{5}, \hat{c}_{6}, \hat{c}_{8}\right\}$ and $c_{\max }:=\min \left\{c_{1}^{*}, \bar{c}_{2}, \bar{c}_{4}, \bar{c}_{6}, \hat{c}_{1}, \hat{c}_{3}, \hat{c}_{7}\right\}$.

Next, suppose that $c=0$ and $\rho \neq 0$. If country $m$ free rides, the embargo equilibrium is characterized by

$$
\begin{aligned}
& e_{f}^{s}=e_{f}^{d}>0 \Longleftrightarrow \rho>-\frac{b+\xi+2\left(2 n^{2}-3 n+1\right) \delta}{n(2 n-1)}=: \hat{\rho}_{1}, \\
& e_{z m}^{s}=e_{m m}^{d}=e_{n m}^{d}<0 \quad \Longleftrightarrow \rho<\frac{b+\xi-2(n-1) \delta}{n}=: \hat{\rho}_{2} .
\end{aligned}
$$

If country $n$ free rides, the embargo equilibrium is characterized by

$$
\begin{aligned}
e_{f}^{s}=e_{f}^{d}>0 & \Longleftrightarrow \rho>-\frac{b+\xi+2\left(2 n^{2}-3 n+1\right) \delta}{2 n^{2}-5 n+2}=: \hat{\rho}_{3}, \\
e_{z m}^{s}=e_{m m}^{d}=e_{n m}^{d}<0 & \Longleftrightarrow \rho<\frac{b+\xi-2(n-1) \delta}{n-2}=: \hat{\rho}_{4} .
\end{aligned}
$$

Observe that $\hat{\rho}_{1}<0$ and $\hat{\rho}_{3}<0$. Moreover, $b+\frac{2 n-1}{2 n^{2}} \xi>2(n-1) \delta$ is sufficient for $\hat{\rho}_{2}>0$ and $\hat{\rho}_{4}>0$. For sufficiently large parameters $\bar{x}$ the domain of the functions $\mathcal{W}^{* h}(0, \rho), \overline{\mathcal{W}}^{f}(0, \rho)$ and $\hat{\mathcal{W}}^{f}(0, \rho)$ is

$$
\mathcal{S}^{\rho}:=\left\{(a, b, \xi, n, \delta, c, \rho) \mid(a, b, \xi, n, \delta, \rho) \in \mathbb{R}_{++}^{6}, c=0, n \geq 2, b+\frac{2 n-1}{2 n^{2}} \xi>2(n-1) \delta \wedge \rho \in\left[\rho_{\min }, \rho_{\max }\right]\right\},
$$

$\operatorname{where}^{26} \rho_{\min }:=\max \left\{-\delta, \bar{\rho}_{2}, \bar{\rho}_{4}, \hat{\rho}_{1}, \hat{\rho}_{3}\right\}$ and $\rho_{\max }:=\min \left\{\bar{\rho}_{1}, \bar{\rho}_{3}, \hat{\rho}_{2}, \hat{\rho}_{4}\right\}$.

${ }^{25} c_{i}^{*}$ and $\bar{c}_{i}$ are derived in Eichner and Pethig (2014b, Appendix B).
${ }^{26} \rho_{i}^{*}$ and $\bar{\rho}_{i}$ are derived in Eichner and Pethig (2014b, Appendix B) 